\title{
Analytical formula for 2D ring artefact suppression
}

DOI:

10.1107/S160057751601479X

\section{Document Version}

Accepted author manuscript

Link to publication record in Manchester Research Explorer

\section{Citation for published version (APA):}

Titarenko, V. (2016). Analytical formula for 2D ring artefact suppression. Journal of Synchrotron Radiation, 23(6), 1447-1461. https://doi.org/10.1107/S160057751601479X

\section{Published in:}

Journal of Synchrotron Radiation

\section{Citing this paper}

Please note that where the full-text provided on Manchester Research Explorer is the Author Accepted Manuscript or Proof version this may differ from the final Published version. If citing, it is advised that you check and use the publisher's definitive version.

\section{General rights}

Copyright and moral rights for the publications made accessible in the Research Explorer are retained by the authors and/or other copyright owners and it is a condition of accessing publications that users recognise and abide by the legal requirements associated with these rights.

\section{Takedown policy}

If you believe that this document breaches copyright please refer to the University of Manchester's Takedown Procedures [http://man.ac.uk/04Y6Bo] or contact uml.scholarlycommunications@manchester.ac.uk providing relevant details, so we can investigate your claim.

\section{OPEN ACCESS}


Journal of Synchrotron Radiation

ISSN 0909-0495

\title{
Analytical formula for 2D ring artefact suppression
}

\author{
Valeriy Titarenko * \\ Henry Moseley X-ray Imaging Facility, Photon Science Institute, Alan Turing building, The University of \\ Manchester, Manchester, M13 9PL UK. Correspondence e-mail: valeriy.titarenko@manchester.ac.uk
}

\begin{abstract}
Ring artefacts are the most disturbing artefacts when reconstructed volumes are segmented. A lot of effort has already been put into better x-ray optics, scintillators and detectors in order to minimise appearance of these artefacts. However, additional processing is often required after standard flat-field correction. Several methods exist to suppress artefacts. One group of methods is based on minimisation of Tikhonov functional. An analytical formula for processing of a single sinogram was developed. In this paper a similar approach is used and a formula for processing 2D projections is found. Thus suppression of ring artefacts is organised as a 2D convolution of "averaged" projections with a given filter. Several approaches are discussed in order to find elements of the filter in a faster and accurate way. Examples of experimental datasets processed by the proposed method are considered.
\end{abstract}

(C) 0000 International Union of Crystallography Printed in Singapore - all rights reserved the Be window on to a recording device (usually CCD (chargecoupled device) or CMOS (complementary metal-oxide semiconductor) cameras) behaves in a similar way. On the other hand, a scintillator, optical lenses and detector also have some dust/dirt and defects on their surfaces. All these lead to the fact that a background image has (at least) two components/profiles: one is stable and the other is slightly moving/stretching during data acquisition. Therefore the standard flat field correction procedure defined by (1) leaves some artefacts on projections which in turn lead to so-called ring artefacts on reconstructed slices, i.e. concentric circles or arches. Some attempts to compensate intensity variations due to beam/monochroamtor instabilities have been made in (Titarenko et al., 2010d). \& Slaney, 2001; Kalender, 2006; Warnett et al., 2016). To perform a reconstruction of a specimen one needs to find optical paths by the formula

$$
P=\ln \frac{I_{\text {flat }}-I_{\mathrm{dark}}}{I-I_{\mathrm{dark}}}
$$

where $I_{\text {flat }}, I_{\text {dark }}$ and $I$ are flat/dark field images and a projection respectively. Note the flat and dark field images should theoretically be taken at the same moment of time when the projection is recorded. Obviously, it is not possible in practical applications, so dark and flat field images are usually taken before and after all projections are acquired.

For many beamlines an x-ray beam may slightly move during an experiment. For instance, beam oscillations and linear move in vertical direction on beamline 05B1-1 at the Canadian Light Source are discussed in (Hinebaugh et al., 2012). For tomography beamlines these fluctuations often lead to flat field image being changed during data acquisition. This effect can be explained in the following way. X-rays travel in a high vacuum tube protected by a beryllium window just before entering an optical hutch where experiments are performed. Any small variation of the beam position and thermal instabilities at a monochromator force intensity profile of the beam to move and stretch/shrink in vertical direction. As the result projection of
Unfortunately, defects on optical components behave differently. If there is an x-ray absorbing feature on a scintillator, then it usually affects those areas on recorded images, which are projections of the feature onto the detector. Thus after the standard flat-field correction has been performed, the corresponding ring artefacts seen after reconstruction are regular, i.e. the circles/arches have same intensity along the whole curve. However, if a scintillator has scratches or dust on its surface (see Fig. 11), then an additional visible light comes from these defects and depends on a total flux of x-rays incident on a wider area around these defects. One can change position of slits in order to use smaller shape of the X-ray beam and still be able to see these bright defects at areas with no x-rays, see Fig 2 As the result for dense materials or anisotropically attenuated specimens one can see intensity of ring artefacts dependent on angle of rotation. Some examples can be found in (Titarenko et al., 2011). Of course, if defects on a scintillator are too strong and/or exposure time to record an image is too long, then some pixels of the detector may become saturated, so we do not have any information from them. 


\section{research papers}

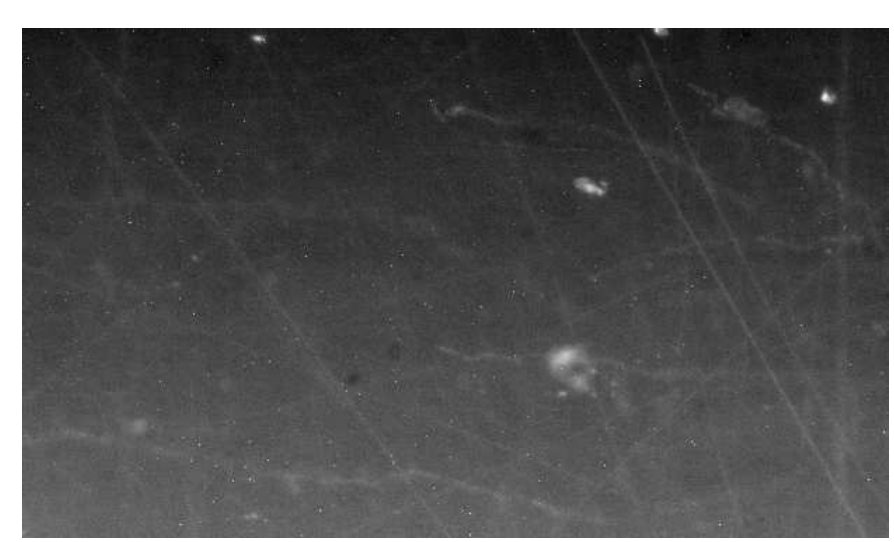

\section{Figure 1}

A $620 \times 360$ region of a flat field image acquired at beamline I12 of Diamond Light Source. Random high intensity pixels and bright structures from defects on a scintillator are seen.

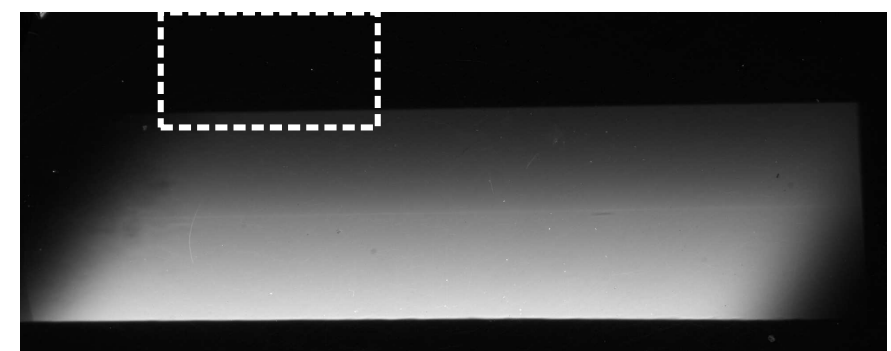

\section{(a)}

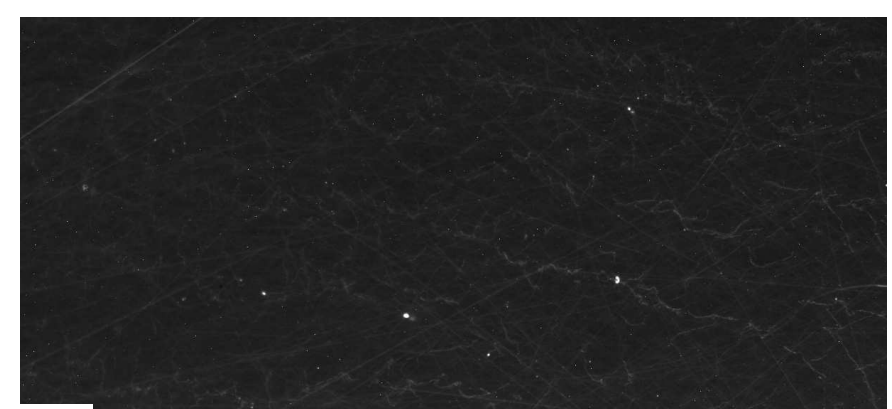

\section{(b)}

\section{Figure 2}

A flat field image ( $a$ ) and its dark part with increased contrast $(b)$ acquired at beamline I12 of Diamond Light Source.

Several methods for ring artefact suppression have already been developed. In principle they can be placed into three main groups. The first group of methods uses a "hardware" solution by usually moving a detector with respect to projections. Thus if there is a defect on a scintillator or Be window then it is either randomly moved to different pixels or blurred (Davis \& Elliott, 1997; Davis et al., 2013). In this case no high intensity rings can be seen on a reconstructed image. However if a defect is strong, then one should expect to see a "wave" rather than a ring on slices. The second group is related to preprocessing sinograms before reconstruction is performed by applying various linear or non-linear filters. Methods from the third group process data after reconstruction and usually remap a slice in polar coordinates, so rings are transformed into stripes. Basically methods from these two groups are similar. A detailed description of some of these methods and their comparison for various data sets can be found in (Sijbers \& Postnov, 2004; Prell et al., 2009; Münch et al., 2009; Titarenko et al., 2010b; Sadi et al., 2010; Titarenko et al., 2010c; Rashid et al., 2012; Brun et al., 2013; Wei et al., 2013; Altunbas et al., 2014; Miqueles et al., 2014; Paleo \& Mirone, 2015; Wolkowski et al., 2015; Baek et al., 2015).

In this paper a new method for preprocessing sinograms/projections is presented. This method is a generalisation of a method from (Titarenko et al., 2010b), which was already modified to be used for irregular ring artefacts (Titarenko et al., 2010c; Titarenko et al., 2011) and is a part of reconstruction software used at beamlines I12 and I13 of Diamond Light Source (Drakopoulos et al., 2015; Pešić et al., 2013). The original method was designed to process each sinogram independently, i.e. to be applied for 1D projections, and assumes "averaged" projections should not have sharp peaks. The same idea of smoothness is used in the proposed algorithm but applied to 2D projections. Modern reconstruction algorithms usually exploit parallelisation provided by GPUs (Graphics Processing Unit) and tend to process several slices at the same time. Thus it is more effective to process chunks of projections rather than individual rows. In case of cone beam geometry this approach is already used. Therefore processing 2D projections may speed up other reconstruction steps by avoiding unnecessary movement or remapping of data. At the same time additional prior information from neighbouring rows of projections should lead to better suppression of artefacts. Note the solution provided by the original method from (Titarenko et al., 2010b) is a vector obtained by multiplying a given matrix by an input vector. In the proposed method a solution (matrix) is a convolution of an input matrix with a 2D filter (matrix), so can be implemented in more efficient way if Fast Fourier transform is used. The proposed $2 \mathrm{D}$ version of the method is a generalisation of the 1D method published by the author (Titarenko, 2016). In the paper performance advantages of the filtering approach versus the standard approach based on the inverse matrix from (Titarenko et al., 2010b) are shown.

In the next section a brief mathematical description of steps to get main formula is provided. Then examples of implementation of the proposed filter are presented followed by discussion. All proofs and strict mathematical formulas can be found in Appendixes.

\section{Brief mathematical formulation}

\subsection{Tikhonov functional}

Let there be an $m \times n$ matrix $A$, a known $m$-vector $u$ and an unknown $n$-vector $z$. We want to solve the following system of 
linear equations

$$
A z=u, \quad \text { or } \quad \sum_{j=1}^{n} A_{i j} z_{j}=u_{i}
$$

Instead of exact matrix $A$ and right-hand side $u$ we are given approximate $A^{h}$ and $u^{\delta}$, where $h$ and $\delta$ errors. For instance $\sum_{i=1}^{m} \sum_{j=1}^{n}\left|A_{i j}^{h}-A_{i j}\right|^{2} \leq h^{2}, \sum_{i=1}^{m}\left|u_{i}^{\delta}-u_{i}\right|^{2} \leq \delta^{2}$. A solution of $A^{h} z=u^{\delta}$ is defined as $z^{h \delta}$. We aim to find an exact solution of (2) knowing $A^{h}, u^{\delta}, h$ and $\delta$. Thus we get a classical inverse problem. The simplest way to estimate solution $z$ is to find an approximate solution $z^{h \delta}$. One may hope that if errors $h$ and $\delta$ tend to zero, then the corresponding solution $z^{h \delta}$ becomes closer to $z$. Unfortunately, for most inverse problems this is not the case as they are usually so-called ill-posed problems. This means that solutions $z$ or $z^{h \delta}$ may not

- exist for the pairs $(A, u)$ and/or $\left(A^{h}, u^{\delta}\right)$,

- be unique,

- be stable with respect to small variations.

To overcome these issues Tikhonov proposed an approach based on minimisation of the following smoothing functional

$$
M^{\alpha}[z]=\sum_{i=1}^{m}\left(\sum_{j=1}^{n} A_{i j}^{h} z_{j}-u_{i}^{\delta}\right)^{2}+\alpha \sum_{j=1}^{n} z_{j}^{2}
$$

for a given parameter $\alpha>0$. Now $M^{\alpha}[z]$ is often called Tikhonov functional. It was shown that the corresponding minimizers $z^{\alpha}$ of (3) tend to the exact solution $z$ when errors $h$ and $\delta$ approach zero. Several methods how to choose $\alpha$ as a function of $h$ and $\delta$ have been proposed. More details on how to solve ill-posed problems can be found in (Tikhonov \& Arsenin, 1977; Tikhonov et al., 1995; Engl et al., 1996).

\subsection{Optimisation problem}

In order to better understand how the theory of inverse and ill-posed problems can be applied to correct ring artefacts we consider the following continuous problem. Suppose there is a $2 \mathrm{D}$ rectangular sensor, so we are able to record an intensity of $\mathrm{x}$-rays incident onto this sensor, i.e. for $\xi \in\left[0, L_{1}\right]$ and $\eta \in\left[0, L_{2}\right]$, where $\xi$ and $\eta$ are the horizontal and vertical coordinates. As a sample is rotated around its vertical axis we may also record intensities as a function of angle $\theta$ (or time $t$ ). For a parallel beam we usually assume that $\theta$ is from 0 to $180^{\circ}$ (or $\pi$ radians). After the standard flat-field correction procedure (1) we get continuous function $\tilde{p}(\xi, \eta, \theta)$. If there are no errors in our data acquisition system the flat-field correction procedure should provide us with an exact function $p(\xi, \eta, \theta)$. In real world a function $q(\xi, \eta, \theta)=p(\xi, \eta, \theta)-\tilde{p}(\xi, \eta, \theta)$ may not be zero. Of course, we try to organise data acquisition in a such way, so possible imperfections of our system do not affect quality of data. Therefore we may always assume that function $q(\xi, \eta, \theta)$ is small with respect to $p(\xi, \eta, \theta)$. So we want the following integral

$$
\int_{0}^{\pi} \int_{0}^{L_{2}} \int_{0}^{L_{1}} q^{2}(\xi, \eta, \theta) d \xi d \eta d \theta
$$

to tend to a small value (ideally zero). In a simplest case we may think that the error does not depend on angle of rotation, i.e. we have $2 \mathrm{D}$ function $q(\xi, \eta)$ and for a given recorded values $\tilde{p}(\xi, \eta, \theta)$ we should find an unknown values $p(\xi, \eta, \theta)$ such that

$$
\int_{0}^{L_{2}} \int_{0}^{L_{1}} q^{2}(\xi, \eta, \theta) d \xi d \eta
$$

or equivalently

$$
\int_{0}^{L_{2}} \int_{0}^{L_{1}}[\tilde{p}(\xi, \eta, \theta)-p(\xi, \eta, \theta)]^{2} d \xi d \eta
$$

are small. At the same time we have additional, so-called a priori information that unknown function $p(\xi, \eta, \theta)$ is smooth at least along $\xi$ and $\eta$ coordinates (Titarenko et al., 2010a). Thus we aim to find such $p(\xi, \eta, \theta)$ that

$$
\int_{0}^{L_{2}} \int_{0}^{L_{1}}\left[\left(\frac{\partial p}{\partial \xi}(\xi, \eta, \theta)\right)^{2}+\left(\frac{\partial p}{\partial \eta}(\xi, \eta, \theta)\right)^{2}\right] d \xi d \eta
$$

has a minimal value.

\subsection{Original $1 \mathrm{D}$ problem}

In the original method, see (Titarenko et al., 2010b), a sinogram $\tilde{p}_{k j}$ is given, $1 \leq k \leq m, 1 \leq j \leq n$, where $m$ and $n$ are the number of rows (projections) and columns (pixels in one row). It is assumed the same unknown value $q_{j}$ should be added to each element of the $j$-th column in order to find the exact sinogram. For a regularisation parameter $\gamma$ Tikhonov functional can be written as

$$
M^{\gamma}=\sum_{k=1}^{m} \sum_{j=1}^{n-1}\left(\tilde{p}_{k j}-\tilde{p}_{k, j+1}+q_{j}-q_{j+1}\right)^{2}+\gamma m \sum_{j=1}^{n} q_{j}^{2} .
$$

Note that Tikhonov functional can be written in several equivalent forms, for each of them different regularisation parameters can be used. In order to separate results obtained for 1D and 2D cases we use $\gamma$ for 1D and $\alpha$ for 2D problems. If we average the sinogram along columns, i.e. use $p_{j}=\frac{1}{m} \sum_{k=1}^{m} \tilde{p}_{k j}$, then the above problem leads to minimisation of

$$
\sum_{j=1}^{n-1}\left(p_{j}-p_{j+1}+q_{j}-q_{j+1}\right)^{2}+\gamma \sum_{j=1}^{n} q_{j}^{2}
$$

and analytical formula to find $q_{j}$ is presented. In (Titarenko et al., 2011) weights $w_{k}$ are used to find "averaged" values $p_{j}=\sum_{k=1}^{m} w_{k} \tilde{p}_{k j}$, so problems to correct for ring artifacts due to nonuniform stripes in sinograms can be solved.

\subsection{Mathematical formulation of $2 \mathrm{D}$ problem}

In case of two-dimensional projections we can write down Tikhonov functional in a similar way. Suppose we are given an "averaged" projection by adding up projections with some weights. Let this "averaged" projection be a rectangular matrix $P$ with elements $p_{i j}, i=1, \ldots, m, j=1, \ldots, n$. This matrix contains some noisy data and we want to find an unknown exact matrix $Z$ with elements $z_{i j}$ such that the respective values of $z_{i j}$ 


\section{research papers}

are close to $p_{i j}$. This approach is similar to the 1D case if we use $q_{i j}=p_{i j}-z_{i j}$, however the choice to use $z_{i j}$ instead of $q_{i j}$ is more convenient for $2 \mathrm{D}$ case due to simpler description for matrices defined below. Our goal is to find matrix $Z$ such that its values $z_{i j}$ are close to values $p_{i j}$ of the given averaged projection and use some additional information about $Z$. We suppose the neighbouring values of $Z$ have similar values, it is a natural assumption, see (Titarenko et al., 2010a). Then for a regularisation parameter $\alpha>0$ Tikhonov functional can be written as

$$
\begin{aligned}
M^{\alpha} & =\frac{1}{2} \sum_{k=1}^{n} \sum_{l=1}^{m}\left(p_{k l}-z_{k l}\right)^{2} \\
+ & \frac{\alpha}{2}\left\{\sum_{k=1}^{n-1} \sum_{l=1}^{m}\left(z_{k l}-z_{k+1 l}\right)^{2}+\sum_{k=1}^{n} \sum_{l=1}^{m-1}\left(z_{k l}-z_{k l+1}\right)^{2}\right\} .
\end{aligned}
$$

To distinguish regularisation parameters used in the original and proposed methods we use different symbols, $\gamma$ and $\alpha$. Comparing formulas (8) and (10) one can see $\alpha \sim 1 / \gamma$. The necessary condition to attain the minimum is that all first derivatives of $M^{\alpha}$ equal zero, i.e.

$$
\frac{\partial M^{\alpha}}{\partial z_{k l}}=\sum_{\hat{k}=1}^{n} \sum_{\hat{l}=1}^{m} A_{k l, \hat{k} \hat{l}} z_{\hat{k} \hat{l}}-p_{k l}=0,
$$

where matrix $A$ is defined below.

For convenience instead of $(n \times m)$-matrices $P$ and $Z$ we use $n m$-vectors $p$ and $z$ by stitching rows. If we define $i=$ $(k-1) m+l, j=(\hat{k}-1) m+\hat{l}, N=n \cdot m$, then

$$
\frac{\partial M^{\alpha}}{\partial z_{i}}=\sum_{j=1}^{N} A_{i j} z_{j}-p_{i}=0
$$

The elements of matrix $A$ can be found in Appendix $\mathrm{A}$. For example, we obtain $(12 \times 12)$-matrix $A$ as

$A=\left(\begin{array}{cccccccccccc}1+2 \alpha & -\alpha & 0 & 0 & -\alpha & 0 & 0 & 0 & 0 & 0 & 0 & 0 \\ -\alpha & 1+3 \alpha & -\alpha & 0 & 0 & -\alpha & 0 & 0 & 0 & 0 & 0 & 0 \\ 0 & -\alpha & 1+3 \alpha & -\alpha & 0 & 0 & -\alpha & 0 & 0 & 0 & 0 & 0 \\ 0 & 0 & -\alpha & 1+2 \alpha & 0 & 0 & 0 & -\alpha & 0 & 0 & 0 & 0 \\ -\alpha & 0 & 0 & 0 & 1+3 \alpha & -\alpha & 0 & 0 & -\alpha & 0 & 0 & 0 \\ 0 & -\alpha & 0 & 0 & -\alpha & 1+4 \alpha & -\alpha & 0 & 0 & -\alpha & 0 & 0 \\ 0 & 0 & -\alpha & 0 & 0 & -\alpha & 1+4 \alpha & -\alpha & 0 & 0 & -\alpha & 0 \\ 0 & 0 & 0 & -\alpha & 0 & 0 & -\alpha & 1+3 \alpha & 0 & 0 & 0 & -\alpha \\ 0 & 0 & 0 & 0 & -\alpha & 0 & 0 & 0 & 1+2 \alpha & -\alpha & 0 & 0 \\ 0 & 0 & 0 & 0 & 0 & -\alpha & 0 & 0 & -\alpha & 1+3 \alpha & -\alpha & 0 \\ 0 & 0 & 0 & 0 & 0 & 0 & -\alpha & 0 & 0 & -\alpha & 1+3 \alpha & -\alpha \\ 0 & 0 & 0 & 0 & 0 & 0 & 0 & -\alpha & 0 & 0 & -\alpha & 1+2 \alpha\end{array}\right)$

for $n=3$ and $m=4$. To find the solution of (12) we want to invert matrix $A$, so $z=A^{-1} p$. These days images from $\mathrm{x}$ ray tomography usually have size about $2000 \times 2000$ pixels. So $N=4,000,000$ and it is very hard to invert $(N \times N)$ matrix $A$ even if it is a sparse matrix. It is shown in Appendix A that for $n=m>3$ the matrix has (at least) two eigenvalues $\lambda=1$ and $1+4 \alpha$. Therefore the condition number
$\kappa(A)=\lambda_{\max } / \lambda_{\min } \geq 1+4 \alpha$, so for large values $\alpha$ the condition number is large and numerical matrix inversion becomes unstable with respect to small errors in data. Storing the inverse matrix may also be an issue due to its size. Therefore we try the following approximate approach. We find the analytical solution only for the central element of the matrix $Z$ which will be the product of a $(n m \times n m)$-matrix $G$ by the $n m$-vector $p$. This matrix $G$ may be considered as two-dimensional filter, which can be used to find matrices $Z$ from given images $P$ when their size are greater than the size of $G$. As it is shown in next sections elements of filter $G$ decay very fast as we go from the central element to the outer ones (several orders of magnitude). Therefore we may set them to zero from some distance or alternatively we may use a smaller filter $G$. For many practical data sets $(64 \times 64)$-filters work very well. So for all elements of matrix $Z$ inside 32 -element boundary we may always select corresponding $(64 \times 64)$ part of matrix $P$ and convolve it with the $(64 \times 64)$-filter $G$. Or in an alternative approach, we just keep $G$ and $P$ the same power-of-two size, so Fast Fourier transform can be used.

We intend to find the values of the central element of the matrix $Z$, therefore we suppose $n$ and $m$ to be odd numbers. Let us write define $\beta=2+1 / \alpha$, so $\alpha=1 /(\beta-2)$, and define $(m \times m)$-matrix $X$ as

$$
X=\left(\begin{array}{cccccc}
\beta & -1 & 0 & \cdots & 0 & 0 \\
-1 & \beta+1 & -1 & \cdots & 0 & 0 \\
0 & -1 & \beta+1 & \cdots & 0 & 0 \\
\vdots & \vdots & \vdots & \ddots & \vdots & \vdots \\
0 & 0 & 0 & \cdots & \beta+1 & -1 \\
0 & 0 & 0 & \cdots & -1 & \beta
\end{array}\right)
$$

by $I$ we denote the $(m \times m)$ identity matrix. Then the matrix $A$ can be written as the following $(n \times n)$-block matrix

$$
A=\frac{1}{\beta-2}\left(\begin{array}{cccccc}
X & -I & 0 & \cdots & 0 & 0 \\
-I & X+I & -I & \cdots & 0 & 0 \\
0 & -I & X+I & \cdots & 0 & 0 \\
\vdots & \vdots & \vdots & \ddots & \vdots & \vdots \\
0 & 0 & 0 & \cdots & X+I & -I \\
0 & 0 & 0 & \cdots & -I & X
\end{array}\right) .
$$

Let us introduce a function $W_{n}(x)$, where the input parameter is a scalar $x$ and the output is the following $(n \times n)$-matrix

$$
W_{n}(x)=\left(\begin{array}{cccccc}
x & -1 & 0 & \cdots & 0 & 0 \\
-1 & x+1 & -1 & \cdots & 0 & 0 \\
0 & -1 & x+1 & \cdots & 0 & 0 \\
\vdots & \vdots & \vdots & \ddots & \vdots & \vdots \\
0 & 0 & 0 & \cdots & x+1 & -1 \\
0 & 0 & 0 & \cdots & -1 & x
\end{array}\right)
$$

Note that $W_{m}(x)$ is an $(m \times m)$-matrix. Then matrix $A$ can be written as

$$
A=\frac{1}{\beta-2} W_{n}\left(W_{m}(\beta)\right),
$$




\section{research papers}

where $W_{m}(\beta)$ is a matrix and $W_{n}\left(W_{m}(\beta)\right)$ is a matrix function. Therefore the inverse of matrix $A$ is

$$
A^{-1}=(\beta-2) W_{n}^{-1}\left(W_{m}(\beta)\right),
$$

where $W_{n}^{-1}(x)$ is the inverse of matrix $W_{n}(x)$, see (Higham, 2008).

Symmetric matrix $W_{n}(x)$ can be written in the form $Y D Y^{T}$, where $D$ is a diagonal matrix and $Y$ is an orthogonal matrix, i.e. $Y^{-1}=Y^{T}$, where $T$ stands for transpose (Higham, 2008). Therefore the inverse matrix $W^{-1}(x)$ can be written as $Y D^{-1} Y^{T}$. In Appendix we find explicit formulas for $Y, D$ and consequently $W^{-1}(x)$ and $W^{-1}\left(W_{n}(\beta)\right)$. As we only need to find the value of the central element of matrix $Z$, then all matrices $(Z, P$ and filter $G$ ) can be rewritten with respect to their central elements, so instead of $m \times n$ matrix $Z$ we denote such matrix as $\check{Z}$ with elements $z_{i j}, i=-\frac{m-1}{2}, \ldots, \frac{m-1}{2}, j=-\frac{n-1}{2}, \ldots, \frac{n-1}{2}$. Note that $n$ and $m$ are odd numbers. If these numbers are large, then the elements of the filter (matrix) $\breve{G}$ can be written as

$$
\check{G}_{j k}=\frac{\beta-2}{4 \pi^{2}} \int_{-\pi}^{\pi} \int_{-\pi}^{\pi} \frac{\cos j \xi \cos k \eta}{\beta+2(1-\cos \xi-\cos \eta)} d \eta d \xi
$$

$$
\check{G}_{j k}=(1-4 \tau) \tau^{k+j} \sum_{q=0}^{\infty} C_{2 q+j+k}^{q} C_{2 q+j+k}^{q+j} \tau^{2 q}
$$

where $\tau=\alpha /(1+4 \alpha)$. The above formula (or formula (61) is derived in Appendix B. In Appendix $\mathrm{C}$ it is shown how to find the infinite sum numerically with high precision. Some useful properties of matrix $\breve{G}$ are derived in Appendix B.9. Examples of matrices $\breve{G}$ are shown in Fig. 3 . Matrix $\breve{G}$ is symmetrical with respect to central horizontal, vertical and diagonal lines. All elements of the matrix are positive and for each row they monotonically decrease with the column index, see Fig. 4 The sum of all elements of matrix $\breve{G}$ (of infinite width/height) is 1 .
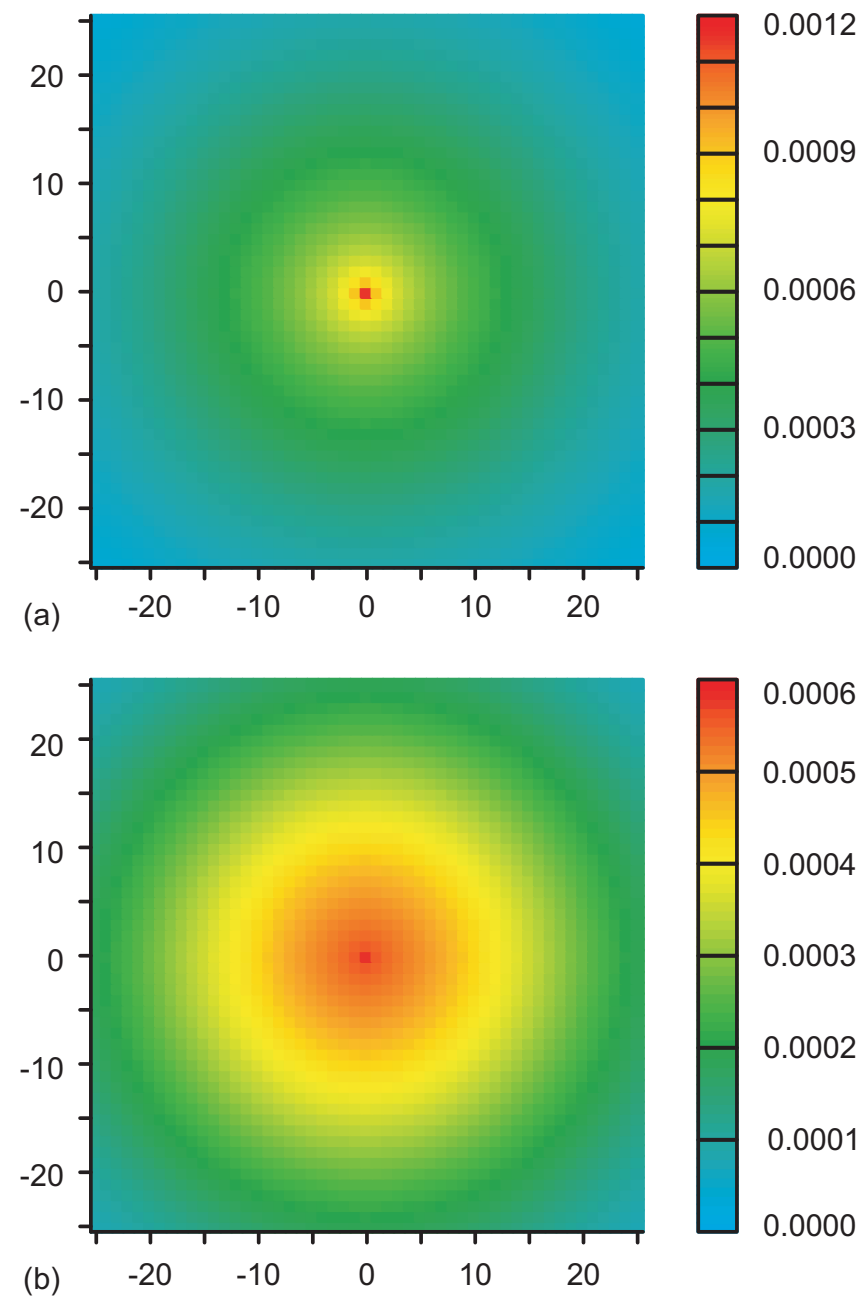

0.0006

0.0005

0.0004

0.0003

0.0002

0.0001

0.0000

Figure 3

The central $51 \times 51$ section of matrix $\breve{G}$ for $(a) \alpha=1000,(b) \alpha=10000$.

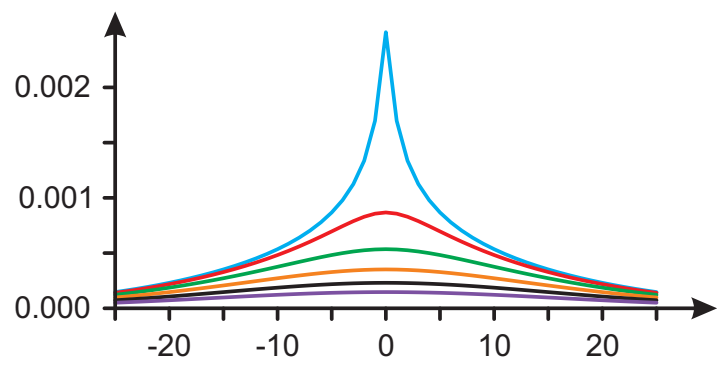

Figure 4

Values of matrix $\check{G}$ found for $\alpha=300$ and rows $0,5,10,15,20,25$ (blue, red, green, orange, black, violet).

Elements of matrix $\breve{G}$ seem to be radial symmetric, however there is a small error between the real values and those found from elements of the central row. If $(i, j)$ is the index of an element of matrix $\check{G}$ and $i, j>0$, then its distance from the central element is $r=\sqrt{i^{2}+j^{2}}$, so we may find an index $k$ such that $k \leq r<k+1$ and use elements $\check{G}_{k-1,0}, \check{G}_{k 0}, \check{G}_{k+1,0}$ and $\check{G}_{k+2,0}$ to approximate value $\check{G}_{i j}$ by cubic interpolation. Relative errors 


\section{research papers}

of such interpolation with respect to the exact element $\check{G}_{i j}$ are shown in Fig. 5 .
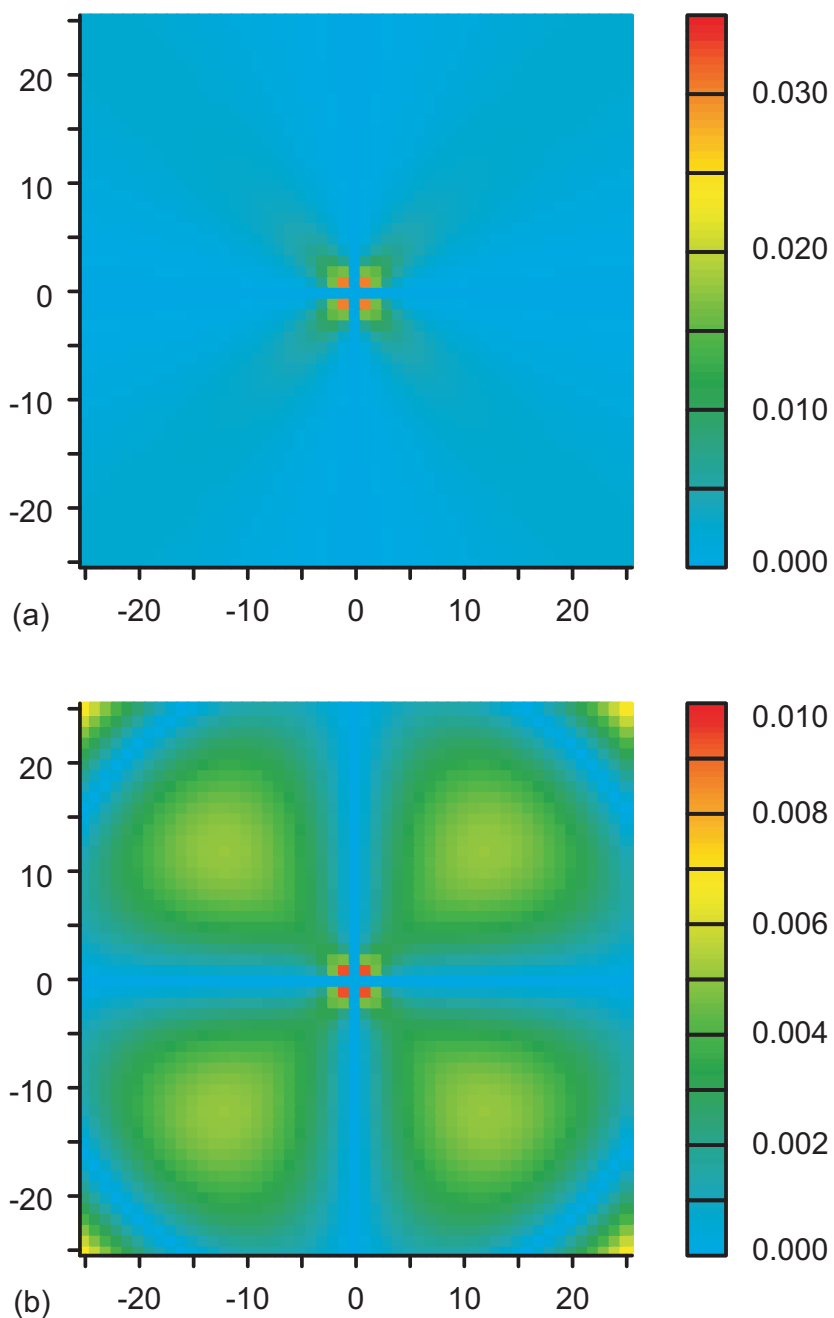

\section{Figure 5}

Relative error of cubic interpolation for $(a) \alpha=100,(b) \alpha=2000$.

If $\alpha=0$ then filtering should not change the input matrix, so all elements of $\breve{G}$ are zeros except the central element, which is 1 . The central element $\breve{G}_{00}$ decreases when $\alpha$ increases, the other elements equal zero for $\alpha=0$, increase, have one maximum and decrease as in Fig. 6 .

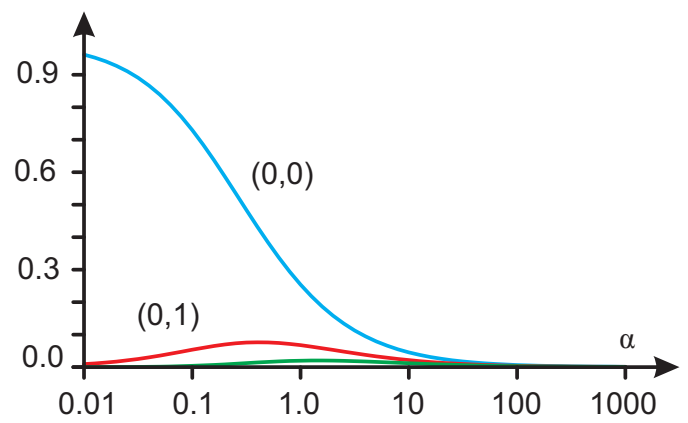

\section{Figure 6}

Values of elements $(0,0),(0,1)$ and $(0,2)$ of matrix $\breve{G}$ as a function of $\alpha$.

\section{Example problems}

The method proposed in this paper is applied to several data sets acquired at Station 16.3 of Synchrotron Radiation Source (SRS), Daresbury Laboratory (second generation synchrotron source, now decommissioned) and beamline I12 of Diamond Light Source (DLS, third generation synchrotron source) in $2008 / 2009$. The choice to use these data sets is dictated by imperfections of an experimental set up those days as these data sets were one of the first test data sets. For instance, in some cases rotation axis was not perfectly vertical, a lot of high intensity peaks were also present on projections as those shown in Fig. 1. A lot of work has been done at I12 to obtain images of sufficiently better quality (Drakopoulos et al., 2015). However these data sets are still useful as similar issues can be seen at other beamlines worldwide. For all data sets a PCO 4000 14bit CCD camera, monochromatic beam and $\mathrm{CdWO}_{4}$ scintillator were used, reconstruction was performed by the software developed by the author for beamline I12.

\subsection{Graphite}

A $5.0 \times 2.5 \mathrm{~mm}$ piece of graphite with some metal particles was scanned at SRS, see Fig. 7 and 8 , This specimen had produced almost ideal regular ring artefacts, even in presence of these dense metal particles. The graphite was slightly tilted and as the result the outer regions of the sample are slightly blurred while the central part is sharp. The ring suppression method is applied for regularisation parameter $\alpha=1000$. 

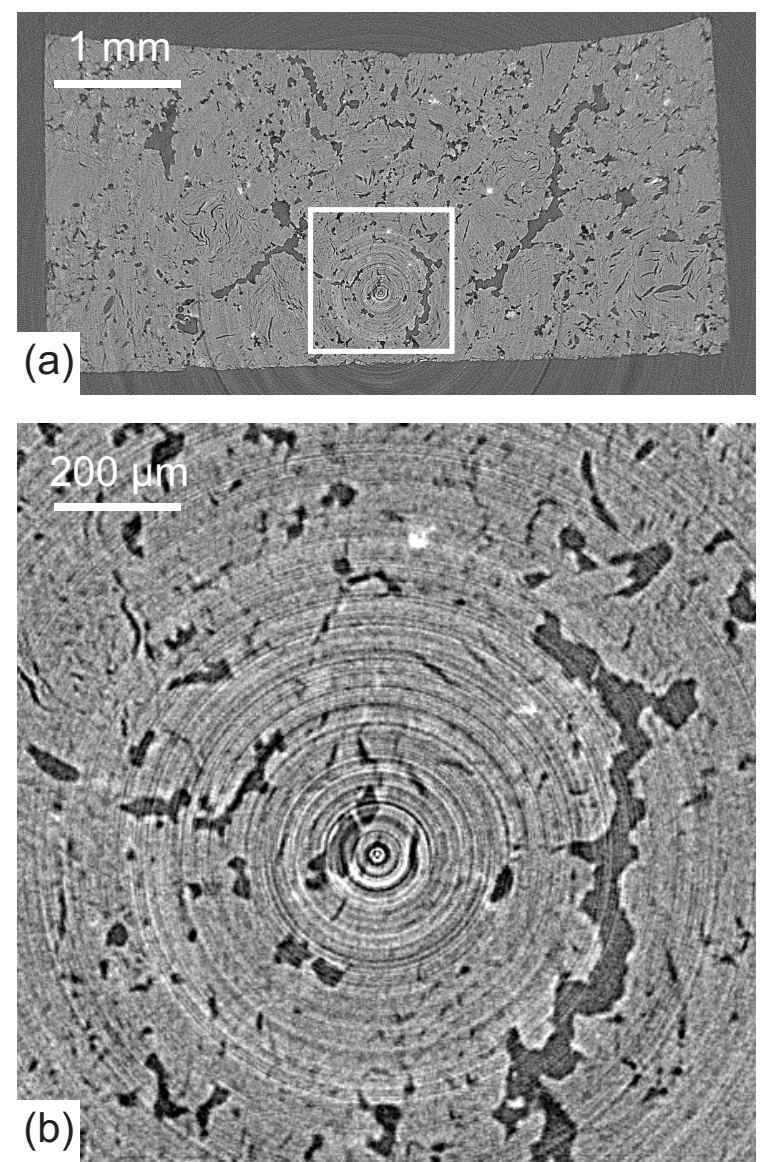

Figure 7

A piece of graphite: (a) the whole section, $(b)$ the central part. No artefact suppression.

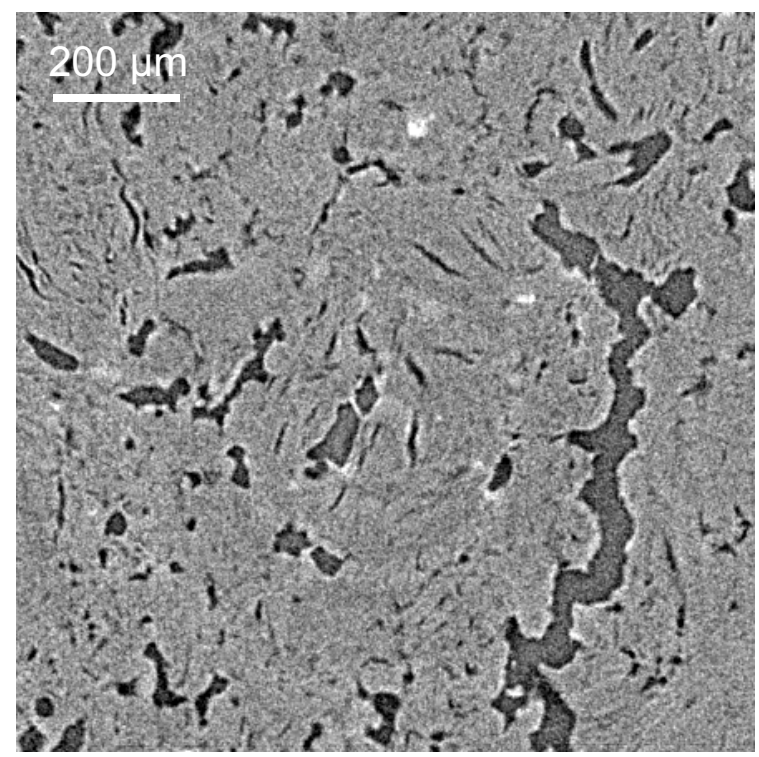

\section{Figure 8}

The central part of the graphite sample after the ring suppression algorithm is applied, $\alpha=1000$.

\subsection{TRISO particles}

A cylindrical container with $1 \mathrm{~mm}$ tristructural-isotropic (TRISO) test particles was also scanned at SRS. A similar specimen was used in (Titarenko et al., 2009) in order to show how a priori information (a known structure of these particles) can be used to remove rings. However this prior information was applied in manual way when corresponding areas of same materials were selected. In this paper an automatic procedure is used.
Beamlines produce almost parallel $\mathrm{x}$-rays, so reconstruction of a sample can be parallelised as all horizontal slices can be found independently of other slices. Therefore many ring artefact suppression algorithms can be used as they usually process 2D slices. However if one has a cone beam geometry or a sample is tilted then several neighbouring sinograms should be used to find one slice. In Fig. 9 the specimen is tilted $\left(0.1^{\circ}\right.$ along the beam and $0.8^{\circ}$ perpendicular to the beam). Therefore it is more efficient to process projections first and then reconstruct slices with a modified filtered backprojection algorithm for tilted samples. 


\section{research papers}
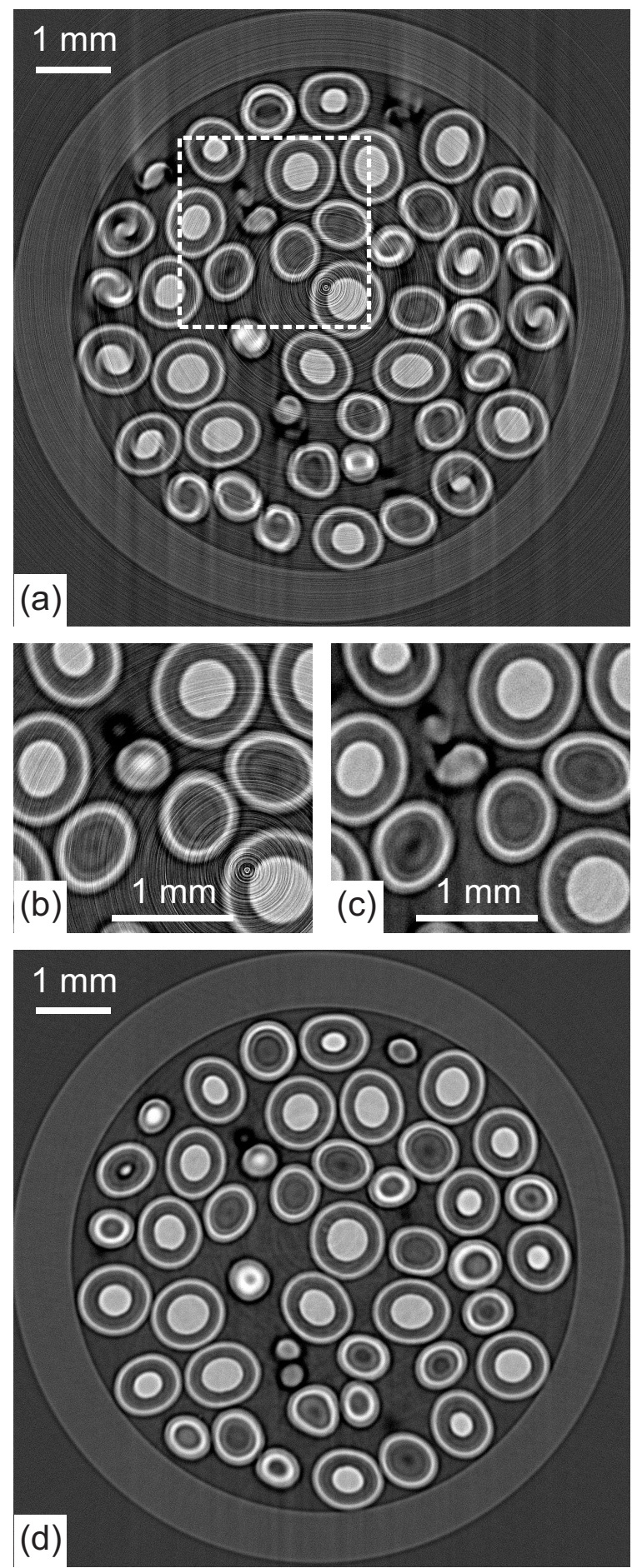

Figure 9

A cylindrical container with TRISO particles, tilted axis of rotation. (a), (b) no ring artefact suppression or tilt correction have been applied, $(c)$ only ring artefacts have been suppressed for $\alpha=10,(d)$ both corrections have been performed.

\subsection{Mouse skull}

A mouse skull was scanned at beamline I12, DLS. Due to scattering a lot of high intensity photons had reached the detector. Identification of the corresponding pixels is easier to per- form on heighbouring projections as 3D neighbouring pixels are used rather than 2D in case of individual sinograms. And a simple correction, e.g. based on linear interpolation unaffected pixels, can be used, see Fig. 10. However due to scratches on the scintillator (similar to those shown in Fig. 2) ring artefact suppression still leaves some rings present after correction.
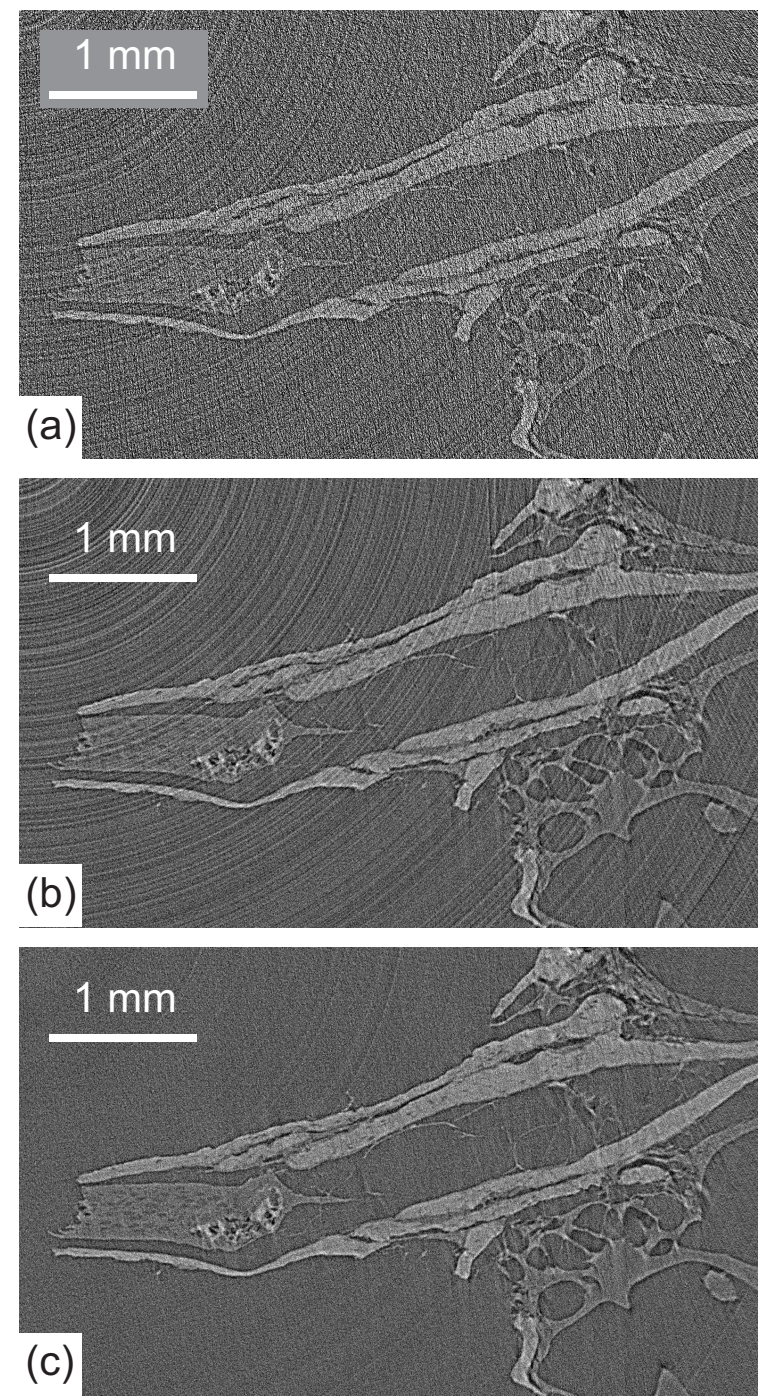

Figure 10

A part of a mouse skull, a lot of high intensity pixels on projections. (a) no correction for high intensity pixels or ring artefacts, $(b)$ high intensity pixels are suppressed, $(c)$ both corrections have been performed, $\alpha=10$.

4.TReactiøial ifmphemention a series, i.e. a sum of infinite terms. Effective numerical implementation is discussed in Appendix C A practical approach is to find the central row of matrix $\breve{G}$ by the formula (64). Then the row above the central one can be found from

$$
\check{G}_{1 k}=\frac{1}{2}\left(\frac{\check{G}_{0 k}}{\tau}-\check{G}_{0 k-1}-\check{G}_{0 k+1}\right)
$$

for $k>0$. And elements of other rows

$$
\check{G}_{j k}=\frac{\check{G}_{j-1, k}}{\tau}-\check{G}_{j-1, k-1}-\check{G}_{j-1, k+1}-\check{G}_{j-2, k},
$$


for $k \geq j$. Suppose we want to find matrix $\check{G}_{j k}$ for $|j|,|k| \leq N$. Then we find the values as it is shown in Fig.11, i.e.

- for $k=0, \ldots, 2 N$ we use (64) to find the central row,

- for $k=1, \ldots, 2 N-1$ we use (20),

- for $j=2, \ldots, N$ and $k=j, \ldots, 2 N-j$, we apply (21),

- for $j=1, \ldots, N, k=0, \ldots, j: \check{G}_{j,-k}=\breve{G}_{-j, k}=$ $\check{G}_{-j,-k}=\check{G}_{k, j}=\check{G}_{k,-j}=\check{G}_{-k, j}=\check{G}_{-k,-j}=\check{G}_{j, k}$.

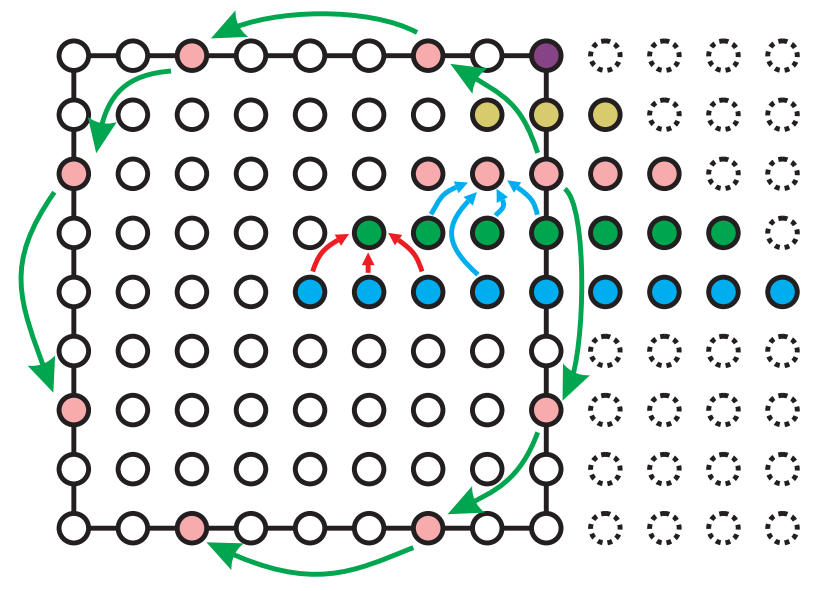

Figure 11

Use of the central row of matrix $\breve{G}$ to find all other elements of the matrix.

5ГHeisDusssitond presented in this paper is in some sense equivalent to the original 1D method presented in Section 2.3 or in (Titarenko et al., 2010b). Thus they both should provide similar results in case of regular ring artefacts when rings' strength does not depend on angle of rotation. For the data sets used as examples the author was able to find values of regularisation parameter $\gamma$ for the original 1D method which gives almost identical images as the proposed method. Of course, in case of irregular rings the result of suppression may depend on a sample. The 1D method designed for irregular rings (Titarenko et al., 2011) can also be used with the 2D method as the original minimisation problem leads to a set of independent problems, which can be solved with the new method.

The main advantage of the $2 \mathrm{D}$ method is that it can be considered as an image filtering procedure. This filtering operation is applied to an "averaged" optical path image and can be used after all projections have been collected. However, this "averaged" image is just a weighted sum of individual optical path images. Therefore this filtering operation can be applied to individual images just after they have been acquired. Taking into account that standard (non-iterative) reconstruction algorithms apply a 1D filter to each projection, then these 1D and 2D filters can be combined in one $2 \mathrm{D}$ filter, i.e. ring suppression can be embedded into a standard reconstruction procedure but with a modified filter.

Implementation of 2D filtering can be done with Fast Fourier transform operations and high performance libraries exist for both CPUs and GPUs.

\section{Appendix A}

\section{Two eigenvalues for matrix $A$}

The matrix $A$ is symmetric and all its non-zero elements are on 5 diagonals $A_{i i}, A_{i, i-1}, A_{i, i+1}, A_{i, i-m}, A_{i, i+m}$. Consider each row of matrix $A$.

1. 3 non-zero elements:
(a) $i=1, A_{i i}=1+2 \alpha, A_{i, i+1}=-\alpha, A_{i, i+m}=-\alpha$;
(b) $i=n, A_{i, i-1}=-\alpha, A_{i i}=1+2 \alpha, A_{i, i+m}=-\alpha$;
(c) $i=n(m-1)+1, A_{i, i-m}=-\alpha, A_{i i}=1+2 \alpha$, $A_{i, i+1}=-\alpha$
(d) $i=n m, A_{i, i-m}=-\alpha, A_{i, i-1}=-\alpha, A_{i i}=1+2 \alpha$;

2. 4 non-zero elements:

(a) $1<i<n, A_{i, i-1}=-\alpha, A_{i i}=1+3 \alpha, A_{i, i+1}=-\alpha$, $A_{i, i+m}=-\alpha$

(b) $i=n+1, n+m+1, \ldots, m(n-2)+1, A_{i, i-m}=-\alpha$, $A_{i i}=1+3 \alpha, A_{i, i+1}=-\alpha, A_{i, i+m}=-\alpha ;$

(c) $i=n+m, n+2 m, \ldots, m(n-1), A_{i, i-m}=-\alpha$, $A_{i, i-1}=-\alpha, A_{i i}=1+3 \alpha, A_{i, i+m}=-\alpha$

(d) $n(m-1)+1<i<n m, A_{i, i-m}=-\alpha, A_{i, i-1}=-\alpha$, $A_{i i}=1+3 \alpha, A_{i, i+1}=-\alpha$

3. 5 non-zero elements (the rest): $A_{i, i-m}=-\alpha, A_{i, i-1}=$ $-\alpha, A_{i i}=1+4 \alpha, A_{i, i+1}=-\alpha, A_{i, i+m}=-\alpha$.

It is clear that the sum of all elements of matrix $A$ for each row is 1 . So if $(n m)$-vector $v=\{1,1, \ldots, 1\}$, then $(A-I) v=0$ and thus $\lambda=1$ is the corresponding eigenvalue.

Now we consider case $n=m>3$ and check that $\lambda=$ $1+4 \alpha$ is another eigenvalue and non-zero elements of one of the corresponding eigenvectors can be written as $v_{i(n-1)}=$ $v_{(i+1)(n-1)+2}=(-1)^{i}, i=1, \ldots, n-1$ and $v_{n}=1, v_{n(n-1)+1}=$ $(-1)^{n}$. For convenience this vector can be represented as an $(n \times n)$-matrix by copying each $n$ consequent elements to a row of the matrix, then it is anti three-diagonal matrix, e.g. for $n=m=5$

$$
v=\left(\begin{array}{ccccc}
0 & 0 & 0 & -1 & 1 \\
0 & 0 & 1 & 0 & -1 \\
0 & -1 & 0 & 1 & 0 \\
1 & 0 & -1 & 0 & 0 \\
-1 & 1 & 0 & 0 & 0
\end{array}\right)
$$

Denote $Q=(A-\lambda I) / \alpha$, then

1. 3 non-zero elements:

(a) $i=1, Q_{i i}=-2, Q_{i, i+1}=-1, Q_{i, i+n}=-1$ and $v_{i}=0, v_{i+1}=0, v_{i+n}=0$;

(b) $i=n, Q_{i, i-1}=-1, Q_{i i}=-2, Q_{i, i+n}=-1$ and $v_{i-1}=-1, v_{i}=1, v_{i+n}=-1$

(c) $i=n(n-1)+1, Q_{i, i-n}=-1, Q_{i i}=-2$, $Q_{i, i+1}=-1$ and $v_{i-n}=-(-1)^{n}, v_{i}=(-1)^{n}$, $v_{i+1}=-(-1)^{n}$; 
(d) $i=n n, Q_{i, i-n}=-1, Q_{i, i-1}=-1, Q_{i i}=-2$ and $v_{i-n}=0, v_{i-1}=0, v_{i}=0$;

2. 4 non-zero elements:

(a) $1<i<n, Q_{i, i-1}=Q_{i i}=Q_{i, i+1}=Q_{i, i+n}=-1$; $v_{i-1}=v_{i}=0, v_{i+1}=-1, v_{i+n}=1($ for $i=n-2)$, $v_{i-1}=0, v_{i}=-1, v_{i+1}=1, v_{i+n}=0($ for $i=n-1)$ and $v_{i-1}=v_{i}=v_{i+1}=v_{i+n}=0$ (otherwise);

(b) $i=n+1,2 n+1, \ldots, n(n-2)+1, Q_{i, i-n}=$ $Q_{i i}=Q_{i, i+1}=Q_{i, i+n}=-1 ; v_{i-n}=v_{i}=0$, $v_{i+1}=(-1)^{n}, v_{i+n}=-(-1)^{n}($ for $i=n(n-3)+1)$, $v_{i-n}=0, v_{i}=-(-1)^{n}, v_{i+1}=0, v_{i+n}=(-1)^{n}$ (for $i=n(n-2)+1), v_{i-n}=v_{i}=v_{i+1}=v_{i+n}=0$ (otherwise);

(c) $i=2 n, 3 n, \ldots, n(n-1), Q_{i, i-n}=Q_{i, i-1}=Q_{i i}=$ $Q_{i, i+n}=-1 ; v_{i-n}=1, v_{i-1}=0, v_{i}=-1, v_{i+n}=0$ (for $i=2 n), v_{i-n}=-1, v_{i-1}=1, v_{i}=v_{i+n}=0$ (for $i=3 n), v_{i-n}=v_{i-1}=v_{i}=v_{i+n}=0$ (otherwise);

(d) $n(n-1)+1<i<n n, Q_{i, i-n}=-1, Q_{i, i-1}=$ $Q_{i i}=Q_{i, i+1}=-1 ; v_{i-n}=0, v_{i-1}=(-1)^{n}$, $v_{i}=-(-1)^{n}, v_{i+1}=0$ (for $\left.i=n(n-1)+2\right)$, $v_{i-n}=(-1)^{n}, v_{i-1}=-(-1)^{n}, v_{i}=v_{i+1}=0$ (for $i=n(n-1)+3), v_{i-n}=v_{i-1}=v_{i}=v_{i+1}=0$ (otherwise);

(e) the rest: $Q_{i, i-n}=Q_{i, i-1}=Q_{i, i+1}=Q_{i, i+n}=-1$; $v_{i-n}=(-1)^{j}, v_{i-1}=-(-1)^{j}, v_{i+1}=(-1)^{j}$, $v_{i+n}=-(-1)^{j}($ for $i=j n+n-j), v_{i-n}=v_{i-1}=$ $0, v_{i+1}=-(-1)^{j}, v_{i+n}=(-1)^{j}($ for $i=j n-j-1)$, $v_{i-n}=(-1)^{j}, v_{i-1}=-(-1)^{j}, v_{i+1}=v_{i+n}=0$ (for $i=j n+2 n-j+1), v_{i-n}=v_{i-1}=v_{i+1}=v_{i+n}=0$ (otherwise).

Now it is easy to check that $\sum_{j=1}^{n^{2}} Q_{i j} v_{j}=0$.

\section{Appendix B Formula for 2D filter}

\section{B.1. Trigonometric formulas}

From the formulas for the sum of sines/cosines we get

$$
\begin{aligned}
& \sin (n \omega+\psi)+\sin ((n-2) \omega+\psi) \\
&=2 \sin ((n-1) \omega+\psi) \cos \omega, \\
& \cos (n+1) x+\cos (n-1) x=2 \cos n x \cos x, \\
& \cos (n-1) x \cos m y+\cos (n+1) x \cos m y \\
&+\cos n x \cos (m-1) y+\cos n x \cos (m+1) y \\
&=2(\cos x+\cos y) \cos n x \cos m y .
\end{aligned}
$$

The following formula is true (Gradshteyn \& Ryzhik, 2015):

$$
\sum_{k=0}^{n-1} \cos (a+k b)=\cos \left(a+\frac{n-1}{2} b\right) \frac{\sin (n b / 2)}{\sin (b / 2)} .
$$

In case of $a=0$ equation (26) gives us

$$
\sum_{k=0}^{n} \cos (k b)=\frac{1}{2}\left(1+\frac{\sin ((2 n+1) b / 2)}{\sin (b / 2)}\right) .
$$

Thus we find Dirichlet sum

$$
1+2 \sum_{k=1}^{n} \cos (k b)=\frac{\sin ((2 n+1) b / 2)}{\sin (b / 2)} .
$$

Some limits for trigonometric functions.

$$
\begin{gathered}
\lim _{x \rightarrow 0} \frac{\sin n x}{\sin x}=n, \\
\lim _{x \rightarrow \pi} \frac{\sin n x}{\sin x}=(-1)^{n+1} n .
\end{gathered}
$$

\section{B.2. Combinatorial formulas}

A factorial $n$ ! of a positive integer number $n$ is defined as $n !=n \cdot(n-1) \cdots 2 \cdot 1,0 ! \equiv 1$, a binomial coefficient $C_{n}^{k}$ can be defined as

$$
C_{n}^{k} \equiv \frac{n !}{k !(n-k) !} .
$$

From the definition $C_{n}^{k}=C_{n}^{n-k}$. The following formulas are true (Benjamin \& Quinn, 2003):

$$
\begin{gathered}
\frac{1}{1-x}=\sum_{n=0}^{\infty} x^{n} \quad \text { for }|x|<1, \\
(x+y)^{n}=\sum_{k=0}^{n} C_{n}^{k} x^{k} y^{n-k}, \\
\sum_{j=0}^{k} C_{m}^{j} C_{n}^{k-j}=C_{m+n}^{k}
\end{gathered}
$$

(Vandermonde convolution/identity).

\section{B.3. Simple integrals}

If $n$ and $m$ are non-negative integer numbers, then for $n \geq m$

$$
\begin{gathered}
\int_{0}^{\pi / 2} \sin ^{2 n+1} x \sin (2 m+1) x d x=\frac{(-1)^{m} \pi}{2^{2 n+2}} C_{2 n+1}^{n-m} \\
\int_{0}^{\pi / 2} \sin ^{2 n} x \cos 2 m x d x=\frac{(-1)^{m} \pi}{2^{2 n+1}} C_{2 n}^{n-m}
\end{gathered}
$$

and the above integrals equal zero for $n<m$, see (Gradshteyn \& Ryzhik, 2015).

Let $f(x)$ and $h(x)$ be odd and even functions respectively, i.e. $f(-x)=-f(x)$ and $h(-x)=h(x)$, then

$$
\begin{gathered}
\int_{-a}^{a} f(x) d x=0, \\
\int_{-a}^{a} h(x) d x=2 \int_{0}^{a} h(x) d x .
\end{gathered}
$$


Taking into account that $\cos \left(x+\frac{\pi}{2}\right)=-\sin x$, we get for nonnegative integer $p$

$$
\int_{0}^{\pi} \cos ^{p} y d y=(-1)^{p} \int_{-\pi / 2}^{\pi / 2} \sin ^{p} x d x .
$$

If $p$ is an odd number, then from (35)

$$
\int_{0}^{\pi} \cos ^{2 n+1} x d x=0
$$

in case of an even $p$ equation (34) can be used and

$$
\int_{0}^{\pi} \cos ^{2 n} x d x=\frac{\pi}{2^{2 n}} C_{2 n}^{n}
$$

In a similar way for $n \geq m$ we obtain

$$
\begin{gathered}
\int_{0}^{\pi} \cos ^{2 n} y \cos 2 m y d y \\
=2(-1)^{m} \int_{0}^{\pi / 2} \sin ^{2 n} x \cos 2 m x d x=\frac{\pi}{2^{2 n}} C_{2 n}^{n-m} \\
\int_{0}^{\pi} \cos ^{2 n+1} y \cos 2 m y d y=0 \\
\int_{0}^{\pi} \cos ^{2 n} y \cos (2 m+1) y d y=0, \\
\int_{0}^{\pi} \cos ^{2 n+1} y \cos (2 m+1) y d y \\
=2(-1)^{m} \int_{0}^{\pi / 2} \sin ^{2 n+1} x \sin (2 m+1) x d x \\
=\frac{\pi}{2^{2 n+1}} C_{2 n+1}^{n-m} .
\end{gathered}
$$

The above integrals are zeros in case of $n<m$.

Dirac $\delta$-function can be written as the following sum

$$
\delta(x)=\frac{1}{\pi} \lim _{n \rightarrow \infty} \frac{\sin n x}{x} .
$$

\section{B.4. The first sequence}

Suppose there is a number $\rho,|\rho|<\frac{1}{4}$ and for integer $n \geq 0$ we want to find

$$
T_{n}=\int_{0}^{\pi} \int_{0}^{\pi} \frac{\cos n x}{1-2 \rho(\cos x+\cos y)} d x d y .
$$

As $\cos x \leq 1, \cos y \leq 1$, then $1-2 \rho(\cos x+\cos y)>0$, so $T_{n}$ is not a singular integral. From (30) we get

$$
\begin{gathered}
T_{n}=\sum_{k=0}^{\infty}(2 \rho)^{k} U_{n k}, \\
U_{n k} \equiv \int_{0}^{\pi} \int_{0}^{\pi} \cos n x(\cos x+\cos y)^{k} d x d y .
\end{gathered}
$$

Taking into account the binomial theorem (31) we get

$$
U_{n k}=\sum_{m=0}^{k} C_{k}^{q} \int_{0}^{\pi} \cos ^{m} x \cos n x d x \int_{0}^{\pi} \cos ^{k-m} y d y .
$$

From (37) and (38) it follows that for even $k=2 \tilde{k}$, we should consider only even $m=2 s$,

$$
U_{n k}=\sum_{s=0}^{\tilde{k}} \frac{\pi}{2^{2(\tilde{k}-s)}} C_{2 \tilde{k}}^{2 s} C_{2(\tilde{k}-s)}^{\tilde{k}-s} \int_{0}^{\pi} \cos ^{2 s} x \cos n x d x
$$

and for odd $k=2 \tilde{k}+1$, we should consider only odd $m=2 s+1$,

$$
U_{n k}=\sum_{s=0}^{\tilde{k}} \frac{\pi}{2^{2(\tilde{k}-s)}} C_{2 \tilde{k}+1}^{2 s+1} C_{2(\tilde{k}-s)}^{\tilde{k}-s} \int_{0}^{\pi} \cos ^{2 s+1} x \cos n x d x .
$$

Due to (39)- (42) we see that $U_{k n}$ is not zero only when both $k$ and $n$ are either even or odd numbers and $2 s \geq n$ or $2 s+1 \geq n$ respectively. So for even $k$ and $n$, i.e. $k=2 \tilde{k}, n=2 \tilde{n}$, we get

$$
\begin{aligned}
U_{n k} & =\frac{\pi^{2}}{2^{k}} \sum_{s=\tilde{n}}^{\tilde{k}} C_{2 \tilde{k}}^{2 s} C_{2(\tilde{k}-s)}^{\tilde{k}-s} C_{2 s}^{s-\tilde{n}}=\frac{\pi^{2}}{2^{k}} C_{2 \tilde{k}}^{\tilde{k}-\tilde{n}} \sum_{s=\tilde{n}}^{\tilde{k}} C_{\tilde{k}-\tilde{n}}^{\tilde{k}-s} C_{\tilde{k}+\tilde{n}}^{\tilde{k}-s} \\
& =\frac{\pi^{2}}{2^{k}} C_{2 \tilde{k}}^{\tilde{k}-\tilde{n}} \sum_{j=0}^{\tilde{k}-\tilde{n}} C_{\tilde{k}-\tilde{n}}^{j} C_{\tilde{k}+\tilde{n}}^{\tilde{k}-\tilde{n}-j}=\frac{\pi^{2}}{2^{k}}\left(C_{2 \tilde{k}}^{\tilde{k}-\tilde{n}}\right)^{2}
\end{aligned}
$$

where the Vandermode identity (32) is used, and for odd $k$ and $n$, i.e. $k=2 \tilde{k}+1, n=2 \tilde{n}+1$

$$
U_{n k}=\frac{\pi^{2}}{2^{k}} \sum_{s=\tilde{n}}^{\tilde{k}} C_{2 \tilde{k}+1}^{2 s+1} C_{2(\tilde{k}-s)}^{\tilde{k}-s} C_{2 s+1}^{s-\tilde{n}}=\frac{\pi^{2}}{2^{k}}\left(C_{2 \tilde{k}+1}^{\tilde{k}-\tilde{n}}\right)^{2} .
$$

So instead of (46) and 47) we get one formula when $n$ and $k$ are both even or odd:

$$
U_{n k}=\frac{\pi^{2}}{2^{k}}\left(C_{k}^{\frac{k-n}{2}}\right)^{2}
$$

$U_{n k}=0$ for $n<k$ or odd $(k-n)$. We replace $k=n+2 q$ in (45)

$$
T_{n}=\pi^{2} \rho^{n} \sum_{q=0}^{\infty}\left[\rho^{q} C_{n+2 q}^{q}\right]^{2}
$$

\section{B.5. The second sequence}

The integral (44) depends only on one integer parameter $n$. Now we consider

$$
S_{n m}=\int_{0}^{\pi} \int_{0}^{\pi} \frac{\cos n x \cos m y}{1-2 \rho(\cos x+\cos y)} d x d y,
$$

which depends on two integer parameters $n$ and $m$. Due to properties of cosine function and symmetry of $S_{n m}$ it is enough to consider only case of $0 \leq m \leq n$ as $S_{n m}=S_{m n}=S_{-n m}=$ $S_{n,-m}$. Note that

$$
\frac{(\cos x+\cos y)}{1-2 \rho(\cos x+\cos y)}=\frac{1}{2 \rho}\left(\frac{1}{1-2 \rho(\cos x+\cos y)}-1\right) .
$$

Let $n, m \neq 0$ at the same time, then

$$
\int_{0}^{\pi} \int_{0}^{\pi} \cos n x \cos m y d x d y=0
$$




\section{research papers}

$$
\int_{0}^{\pi} \int_{0}^{\pi} \frac{(\cos x+\cos y) \cos n x \cos m y}{1-2 \rho(\cos x+\cos y)} d x d y=\frac{1}{2 \rho} S_{n m} .
$$

Therefore if we use identity (25) then

$$
S_{n-1, m}+S_{n+1, m}+S_{n, m-1}+S_{n, m+1}=\frac{S_{n m}}{\rho} .
$$

For $n=m=0$ we obtain

$$
S_{10}=\frac{1}{4}\left(S_{-10}+S_{10}+S_{0,-1}+S_{01}\right)=\frac{S_{00}-\pi^{2}}{4 \rho} .
$$

Taking into account (49) and $S_{n,-1}=S_{n, 1}$, we get

$$
2 S_{n 1}=\frac{S_{n, 0}}{\rho}-S_{n-1,0}-S_{n+1,0}=\frac{T_{n}}{\rho}-T_{n-1}-T_{n+1} .
$$

As $\left(C_{n+2 q}^{q}\right)^{2}-\left(C_{n-1+2 q}^{q}\right)^{2}=0$ for $q=0$, we may write down

$$
\begin{gathered}
\frac{T_{n}}{\rho}-T_{n-1}=\pi^{2} \rho^{n-1} \sum_{q=0}^{\infty} \rho^{2 q}\left[\left(C_{n+2 q}^{q}\right)^{2}-\left(C_{n-1+2 q}^{q}\right)^{2}\right] \\
=\pi^{2} \rho^{n+1} \sum_{s=0}^{\infty} \rho^{2 s}\left[\left(C_{n+2 s+2}^{s+1}\right)^{2}-\left(C_{n+2 s+1}^{s+1}\right)^{2}\right], \\
\frac{S_{n 1}}{\pi^{2} \rho^{n+1}}=\sum_{q=0}^{\infty} \frac{\rho^{2 q}}{2}\left[\left(C_{n+2 q+2}^{q+1}\right)^{2}-\left(C_{n+2 q+1}^{q+1}\right)^{2}-\left(C_{n+2 q+1}^{q}\right)^{2}\right] \\
=\sum_{q=0}^{\infty} \rho^{2 q} C_{n+2 q+1}^{q} C_{n+2 q+1}^{q+1} .
\end{gathered}
$$

Now check that the following formula is true for $0 \leq m \leq n$

$$
S_{n m}=\pi^{2} \rho^{n+m} \sum_{q=0}^{\infty} \rho^{2 q} C_{2 q+n+m}^{q} C_{2 q+n+m}^{q+m} .
$$

We use mathematical induction. Above we have checked the formula for $m=0$ and $m=1$. Assume the formula is true for $0 \leq m<k$. Then

$$
\frac{S_{n-1, k} / \rho-S_{n-1, k-1}-S_{n-2, k}}{\pi^{2} \rho^{n+k-2}}
$$

can be rewritten as Taylor series of $\rho^{2}$ with coefficients

$$
\begin{aligned}
C_{2 q+n+k-1}^{q} C_{2 q+n+k-1}^{q+k}-C_{2 q+n+k-2}^{q} C_{2 q+n+k-2}^{q+k-1} & \\
& -C_{2 q+n+k-2}^{q} C_{2 q+n+k-2}^{q+k}=C_{2 q+n+k-2}^{q-1} C_{2 q+n+k-1}^{q+k}
\end{aligned}
$$

for $q>0$ and zero for $q=0$. Therefore

$$
\begin{aligned}
\frac{S_{n k}}{\pi^{2} \rho^{n+k}} & =\frac{S_{n-1, k} / \rho-S_{n-1, k-1}-S_{n-2, k}-S_{n-1, k+1}}{\pi^{2} \rho^{n+k-2}} \\
& =\sum_{s=0}^{\infty} \rho^{2 s}\left[C_{2 s+n+k}^{s} C_{2 s+n+k+1}^{s+k+1}-C_{2 s+n+k}^{s} C_{2 s+n+k}^{s+k+1}\right] \\
& =\sum_{s=0}^{\infty} \rho^{2 s} C_{2 s+n+k}^{s} C_{2 s+n+k}^{s+k} .
\end{aligned}
$$

\section{B.6. The third sequence}

Let there be $\varphi \in\left(0, \frac{\pi}{2}\right)$ and

$$
V_{n} \equiv \int_{0}^{\pi} \frac{\cos n x}{1-\cos x \sin \varphi} d x
$$

Using formula (24) we obtain for $n>0$

$$
\begin{aligned}
V_{n+1} & +V_{n-1}=\int_{0}^{\pi} \frac{2 \cos x \cos n x}{1-\cos x \sin \varphi} d x \\
& =\frac{2}{\sin \varphi} \int_{0}^{\pi}\left(\frac{1}{1-\cos x \sin \varphi}-1\right) \cos n x d x \\
& =\frac{2}{\sin \varphi} V_{n},
\end{aligned}
$$

since $\int_{0}^{\pi} \cos n x d x=0$. We may find that

$$
V_{0}=\frac{\pi}{\cos \varphi}, \quad V_{1}=\frac{\pi}{\cos \varphi} \frac{\sin \varphi}{1+\cos \varphi}
$$

and therefore use the recurrent formula

$$
V_{n}=\frac{2}{\sin \varphi} V_{n-1}-V_{n-2} .
$$

Since

$$
\frac{2}{\sin \varphi} \frac{\sin \varphi}{1+\cos \varphi}-1=\frac{1-\cos \varphi}{1+\cos \varphi}=\frac{\sin ^{2} \varphi}{(1+\cos \varphi)^{2}},
$$

then $V_{n}$ is a geometric sequence, i.e. $V_{n+1}=\gamma V_{n}$, where

$$
\gamma=\frac{\sin \varphi}{1+\cos \varphi} .
$$

So the values of sequence $V_{n}$ can be written as

$$
V_{n}=\frac{\pi}{\cos \varphi}\left(\frac{\sin \varphi}{1+\cos \varphi}\right)^{n}
$$

\section{B.7. Eigenvalues/eigenvectors}

Let us consider an $(n \times n)$-matrix $B_{n}$

$$
B_{n}=\left(\begin{array}{cccccc}
2 \sigma-1 & -1 & 0 & \cdots & 0 & 0 \\
-1 & 2 \sigma & -1 & \cdots & 0 & 0 \\
0 & -1 & 2 \sigma & \cdots & 0 & 0 \\
\vdots & \vdots & \vdots & \ddots & \vdots & \vdots \\
0 & 0 & 0 & \cdots & 2 \sigma & -1 \\
0 & 0 & 0 & \cdots & -1 & 2 \sigma-1
\end{array}\right)
$$

We aim to find its eigenvalues, therefore we want to solve equation $\operatorname{det} B_{n}=0$. For this purpose we replace the element $(1,1)$ of $B_{n}$ by $2 \sigma$ and denote the corresponding matrix as $F_{n}$. If we use the first row of $B_{n}$ to find its determinant, then $\operatorname{det} B_{n}=(2 \sigma-1) \operatorname{det} F_{n-1}-\operatorname{det} F_{n-2}$. In a similar way we get $\operatorname{det} F_{n}=2 \sigma \operatorname{det} F_{n-1}-\operatorname{det} F_{n-2}$, thus $\operatorname{det} B_{n}=\operatorname{det} F_{n}-\operatorname{det} F_{n-1}$, $\operatorname{det} F_{n}+\operatorname{det} F_{n-2}=2 \sigma \operatorname{det} F_{n-1}$ and

$$
\operatorname{det} B_{n}-\operatorname{det} B_{n-2}=2 \sigma \operatorname{det} B_{n-1} \text {. }
$$

Therefore we need to find a sequence $\left\{b_{n}\right\}$ such that

$$
b_{n}+b_{n-2}=2 \sigma b_{n-1} \text {. }
$$


Of course if one sequence $\left\{b_{n}\right\}$ is found, then $\left\{\tau b_{n}\right\}$ also a solution of the above equation. We want to find $\tau$ such that $\operatorname{det} B_{n}=b_{n}$.

Let $\sigma \in[-1,1]$, then taking into account (23) a solution of (53) is $b_{n}=\tau \sin (n \omega+\psi), \sigma=\cos \omega$. Please note that matrices $B_{n}$ are formally defined for $n \geq 3$. One can find their determinants explicitly for $n=3$ and 4: $\operatorname{det} B_{3}=8 \sigma^{3}-8 \sigma^{2}-2 \sigma+2$, $\operatorname{det} B_{4}=16 \sigma^{4}-16 \sigma^{3}-8 \sigma^{2}+8 \sigma$, so from (53) we get $b_{n}=2 \sigma b_{n+1}-b_{n+2}$ and can extrapolate values $b_{n}$ for $n<3$ : $b_{2}=4 \sigma^{2}-4 \sigma, b_{1}=2 \sigma-2, b_{0}=0$. Thus we get the following system of equations

$$
\begin{aligned}
& b_{0}=\tau \sin (\psi)=0, \\
& b_{1}=\tau \sin (\omega+\psi)=2 \sigma-2 .
\end{aligned}
$$

The first equation is valid if either $\tau=0$ or $\psi=0$ or $\pi$. The case of $\tau=0$ is not possible, since the second equation would always be zero. Since $\sin (\theta+\pi)=-\sin \theta$, then we may only consider the case of $\psi=0$ and change the sign of $\tau$ to get the solution for $\psi=\pi$. Thus

$$
\begin{gathered}
\tau=\frac{2 \sigma-2}{\sin \omega}=\frac{2(\cos \omega-1)}{\sin \omega}, \\
\operatorname{det} B_{n}=2(\cos \omega-1) \frac{\sin n \omega}{\sin \omega} .
\end{gathered}
$$

Now we show that the above equation has $n$ roots, so there is no reason to consider the case of $|\sigma|>1$.

Taking into account (28) we find the first root, $\omega=0$. The other roots can be found from $\sin (n \omega)=0$, so $\omega=k \pi / n$ and $k=0, \ldots, n-1$. Note that due to $k \neq n$. As the result $\operatorname{det} B_{n}=0$ if $\sigma=\cos \frac{k \pi}{n}, k=0, \ldots, n-1$. All eigenvalues of matrix $B_{n}$ can be written as $\lambda_{k}=2\left(\sigma+\rho_{k}\right)$, where $\rho_{k}=\cos \frac{k \pi}{n}$, $k=1, \ldots, n$.

For a given eigenvalue $\lambda_{k}$ we find an eigenvector $y=$ $\left\{y_{1}, \ldots, y_{n}\right\}$. In this paper we consider only the case of odd $n$. We need to solve the system of $n$ linear equations

$$
\left\{\begin{array}{l}
\left(1+2 \rho_{k}\right) y_{1}+y_{2}=0 \\
y_{q-1}+2 \rho_{k} y_{q}+y_{q+1}=0, \quad q=2, \ldots, n-1 \\
y_{n-1}+\left(1+2 \rho_{k}\right) y_{n}=0
\end{array}\right.
$$

Let us denote $\theta=k \pi / 2 n$ and check that for $k=n, \rho_{k}=1$ and

$$
y_{q}=1
$$

is the solution of (54); for $k<n$

$$
y_{q}=(-1)^{q} \sin (2 q-1) \theta .
$$

If $k$ is odd, then $y_{n+1-q}=y_{q}$, i.e. vector $y$ is symmetric. If $k$ is even, then $y_{n+1-q}=-y_{q}$, i.e. vector $y$ is antisymmetric. Thus it is enough to check the case of $q<n / 2$. We get $y_{1}=-\sin \theta$, $y_{2}=\sin 3 \theta=\sin \theta\left(3-4 \sin ^{2} \theta\right)=\sin \theta(2 \cos 2 \theta+1)$, $2 \rho_{k}+1=2 \cos 2 \theta+1$, so the first equation of (54) is true. As

$$
\sin (2 q-3) \theta+\sin (2 q+1) \theta=2 \sin (2 q-1) \theta \cos 2 \theta
$$

then the second equation in (54) is also true.

Now we find the norm of vector $y$. For $k=n$ it is $n$ and for $k<n$

$$
\sum_{q=1}^{n} y_{q}^{2}=\sum_{q=1}^{n} \sin ^{2}(2 q-1) \theta=\frac{1}{2} \sum_{q=1}^{n}(1-\cos 2(2 q-1) \theta) .
$$

Substituting $a=2 \theta$ and $b=4 \theta$ into 26 and taking into account that $\sin 2 n \theta=\sin 2 k \pi=0$ we find that the norm for $k>0$ is $n / 2$. Finally, we get the normalised vector $y$ with elements

$$
y_{q}= \begin{cases}\frac{(-1)^{q-1}}{\sqrt{n / 2}} \sin \frac{(2 q-1) k \pi}{2 n}, & k=1, \ldots, n-1, \\ \frac{1}{\sqrt{n}}, & k=n .\end{cases}
$$

\section{B.8. Inverse matrix $B^{-1}$}

The original matrix $B \equiv B_{n}$ from (52) can be written as

$$
B=Y D Y^{T}
$$

where matrix $Y$ is the orthogonal matrix, i.e. $Y^{-1}=Y^{T}$, with elements

$$
Y_{i j}= \begin{cases}\frac{(-1)^{i-1}}{\sqrt{n / 2}} \sin \frac{(2 i-1) j \pi}{2 n}, & j=1, \ldots, n-1, \\ \frac{1}{\sqrt{n}}, & j=n .\end{cases}
$$

and matrix $D$ is the diagonal one with elements

$$
D_{i i}=2\left(\sigma+\cos \frac{i \pi}{n}\right),
$$

see (Higham, 2008). The inverse of matrix $B$ can then be written as

$$
B^{-1}=Y D^{-1} Y^{T}
$$

where $D^{-1}$ is a diagonal matrix with elements

$$
D_{i i}^{-1}=\frac{1}{2\left(\sigma+\cos \frac{i \pi}{n}\right)} .
$$

Therefore the element $(k, j)$ of matrix $D^{-1} Y^{T}$

$$
\left.D^{-1} Y^{T}\right|_{k j}=\sum_{q=1}^{n} D_{k q}^{-1} Y_{q j}^{T}=D_{k k}^{-1} Y_{k j}^{T}=D_{k k}^{-1} Y_{j k}
$$

and the elements of $B^{-1}$ can be written as

$$
B_{i j}^{-1}=\sum_{k=1}^{n} Y_{i k} D_{k k}^{-1} Y_{j k}
$$

Then

$$
B_{i j}^{-1}=\frac{1}{n}\left[\sum_{k=1}^{n-1} \frac{(-1)^{i+j}}{\sigma+\cos \frac{k \pi}{n}} \sin \frac{(2 i-1) k \pi}{2 n} \sin \frac{(2 j-1) k \pi}{2 n}+\frac{1}{2(\sigma-1)}\right] .
$$

Taking in to account that $\sin \frac{(2 i-1)(2 n-k) \pi}{2 n}=\sin \frac{(2 i-1) k \pi}{2 n}$ and $\cos \frac{n \pi}{n}=-1$ we get

$$
B_{i j}^{-1}=\frac{1}{2 n} \sum_{k=1}^{2 n-1} \frac{(-1)^{i+j}}{\sigma+\cos \frac{k \pi}{n}} \sin \frac{(2 i-1) k \pi}{2 n} \sin \frac{(2 j-1) k \pi}{2 n} .
$$




\section{research papers}

All matrices mentioned above have indices starting from 1 . However our aim is to find a filter to be applied to a matrix. Therefore it may be more convenient to rewrite the same matrices as ones centered with respect to the central element. Since $n$ and $m$ are both odd, this central element always exists and unique. If a standard matrix $Q$ is given, then by $\breve{Q}$ we denote a matrix centered with respect to its central element. So instead of matrix $B_{i j}^{-1}$ defined for $i=1, \ldots, n, j=1, \ldots, n$ we subtract $n$ from $k$ and $(\check{n}+1)$ from the other indices of the above formula, where $\check{n} \equiv(n-1) / 2$, and get

$$
\begin{aligned}
\check{B}_{i j}^{-1} & =\frac{1}{2 n} \sum_{k=-(n-1)}^{n-1} \frac{(-1)^{i+j}}{\sigma+\cos \frac{(k+n) \pi}{n}} \\
& \times \sin \frac{(2(i+\check{n})+1)(k+n) \pi}{2 n} \sin \frac{(2(j+\check{n})+1)(k+n) \pi}{2 n},
\end{aligned}
$$

where $i, j=-\check{n}, \ldots, \check{n}$. We may rewrite

$$
\begin{gathered}
\sin \frac{(2(i+\check{n})+1)(k+n) \pi}{2 n}=(-1)^{i+\check{n}} \sin \left(\frac{i k \pi}{n}+\frac{k+1}{2} \pi\right), \\
\cos \frac{(k+n) \pi}{n}=-\cos \frac{k \pi}{n}, \\
\check{B}_{i j}^{-1}=\frac{1}{2 n} \sum_{k=-(n-1)}^{n-1} \frac{\sin \left(\frac{i k \pi}{n}+\frac{k-1}{2} \pi\right) \sin \left(\frac{j k \pi}{n}+\frac{k-1}{2} \pi\right)}{\sigma-\cos \frac{k \pi}{n}} \\
=\frac{1}{4 n} \sum_{k=-(n-1)}^{n-1} \frac{\cos \frac{(i-j) k \pi}{n}+\cos \frac{(i+j+n) k \pi}{n}}{\sigma-\cos \frac{k \pi}{n}} .
\end{gathered}
$$

For the central row of $\check{B}^{-1}$ we get

$$
\check{B}_{0 j}^{-1}=\frac{1}{4 n} \sum_{k=-(n-1)}^{n-1} \frac{\cos \frac{j k \pi}{n}\left(1+(-1)^{k}\right)}{\sigma-\cos \frac{k \pi}{n}} .
$$

For odd $k$ the value of $\left(1+(-1)^{k}\right)=0$, so we need to consider only even $k$ and use $s=k / 2$ :

$$
\check{B}_{0 j}^{-1}=\frac{1}{2 n} \sum_{s=-\check{n}}^{\check{n}} \frac{\cos \frac{2 j s \pi}{n}}{\sigma-\cos \frac{2 s \pi}{n}} .
$$

\section{B.9. Inversion of the matrix function}

The matrix $W_{n}(x)$ is defined in (16). According to (57) the central row of the function $W_{n}^{-1}(x)$ can be written as

$$
\left.W_{n}^{-1}\right|_{0 j}=\frac{1}{n} \sum_{s=-\check{n}}^{\check{n}} \cos \frac{2 j s \pi}{n}\left(x+1-2 \cos \frac{2 s \pi}{n}\right)^{-1} .
$$

Now we need to find the matrix $W_{n}^{-1}\left(W_{m}(\beta)\right)$. If $x$ and $v$ are two scalars, then $W_{m}(x)+v=W_{m}(x+v)$. Therefore

$$
\left(W_{m}(x)+1-2 \cos \frac{2 s \pi}{n}\right)^{-1}=W_{m}^{-1}\left(x+1-2 \cos \frac{2 s \pi}{n}\right) .
$$

In principle we only need the central row of $(n m \times n m)$ matrix $W_{n}^{-1}\left(W_{m}(\beta)\right)$. Formula (58) can be used to find $n$ $(m \times m)$-matrices which form the central $(m \times n m)$-block of $W_{n}^{-1}\left(W_{m}(\beta)\right)$. So for the $j$-th matrix $\check{R}$ we may write down

$$
\check{R}_{0 k}=\frac{1}{n m} \sum_{s=-\check{n}}^{\check{n}} \sum_{r=-\check{m}}^{\check{m}} \frac{\cos \frac{2 j s \pi}{n} \cos \frac{2 k r \pi}{m}}{\beta+2\left(1-\cos \frac{2 s \pi}{n}-\cos \frac{2 r \pi}{m}\right)},
$$

where $\check{m}=(m-1) / 2$. Therefore the $(j \cdot m+k)$-th element of the central row of the matrix $A^{-1}$ defined in (18) can be written as

$$
\frac{\beta-2}{n m} \sum_{s=-\check{n}}^{\check{n}} \sum_{r=-\check{m}}^{\check{m}} \frac{\cos \frac{2 j s \pi}{n} \cos \frac{2 k r \pi}{m}}{\beta+2\left(1-\cos \frac{2 s \pi}{n}-\cos \frac{2 r \pi}{m}\right)} .
$$

If values $n$ and $m$ are relatively large, then instead of the sum we may consider a two-dimensional integral. So define continuous variables $\xi$ and $\eta$ such that $\xi, \eta \in[-\pi, \pi]$ and $s=\frac{\xi n}{2 \pi}$, $r=\frac{\eta m}{2 \pi}$ and introduce an integral

$$
\check{G}_{j k}=\frac{\beta-2}{4 \pi^{2}} \int_{-\pi}^{\pi} \int_{-\pi}^{\pi} \frac{\cos j \xi \cos k \eta}{\beta+2(1-\cos \xi-\cos \eta)} d \eta d \xi,
$$

then the elements of the central row of matrix $A^{-1}$ tend to $\check{G}_{j k}$ for large $n$ and $m$. We denote $\tau=\alpha /(1+4 \alpha)$ and rewrite the last formula as

$$
\check{G}_{j k}=\frac{1-4 \tau}{\pi^{2}} \int_{0}^{\pi} \int_{0}^{\pi} \frac{\cos j \xi \cos k \eta}{1-2 \tau(\cos \xi+\cos \eta)} d \eta d \xi .
$$

Some useful properties of (59) can be found. As cosine is an even function, we get than $\check{G}_{j k}$ is is symmetrical with respect to horizontal, vertical or diagonal flipping, i.e. $\check{G}_{j k}=\check{G}_{-j k}=$ $\check{G}_{j,-k}=\check{G}_{k j}$. Thus it is enough to consider $k \geq j \geq 0$. Formulas (49) and (50) give us

$$
\begin{gathered}
\check{G}_{j k}=\tau\left(\check{G}_{j-1 k}+\check{G}_{j+1 k}+\check{G}_{j k-1}+\check{G}_{j k+1}\right), \\
\check{G}_{00}=\tau\left(4 \check{G}_{01}+1\right) .
\end{gathered}
$$

In section B.5 it is shown how integral in (59) can be calculated, so

$$
\check{G}_{j k}=(1-4 \tau) \tau^{k+j} \sum_{q=0}^{\infty} C_{2 q+j+k}^{q} C_{2 q+j+k}^{q+j} \tau^{2 q} .
$$

From the above formula we get other properties of $\check{G}_{j k}$ :

- $\check{G}_{j k}>0$, since $\tau<\frac{1}{4}$ and binomial coefficients are nonnegative.

- $\check{G}_{j, k+1}<\check{G}_{j, k}$, since

$$
\begin{aligned}
& \frac{C_{2 q+j+k}^{q}}{C_{2 q+j+k+1}^{q}}=\frac{q+j+k+1}{2 q+j+k+1}>\frac{1}{2} \\
& \frac{C_{2 q+j+k}^{q+j}}{C_{2 q+j+k+1}^{q+j}}=\frac{q+k+1}{2 q+j+k+1}>\frac{1}{2}
\end{aligned}
$$

and therefore

$$
\tau C_{2 q+j+k+1}^{q} C_{2 q+j+k+1}^{q+j}<C_{2 q+j+k}^{q} C_{2 q+j+k}^{q+j}
$$


- The sum $S_{j}$ of all elements of the $j$-th row of matrix $\breve{G}$

$$
\sum_{k=-\infty}^{\infty} \check{G}_{j k}=\sqrt{1-4 \tau}\left(\frac{1-2 \tau-\sqrt{1-4 \tau}}{2 \tau}\right)^{|j|} .
$$

Taking into account formula (27) and the property (43), we obtain

$$
S_{j}=\frac{1-4 \tau}{\pi} \int_{0}^{\pi} \frac{\cos j \xi}{1-2 \tau(\cos \xi+1)} d \xi
$$

If we introduce $\varphi$ such that $\sin \varphi=2 \tau /(1-2 \tau), \cos \varphi=$ $\sqrt{1-4 \tau} /(1-2 \tau)$, then $\varphi \in\left(0, \frac{\pi}{2}\right)$, since $0<\tau<\frac{1}{4}$, and the value of $S_{j}$ follows from the results of section B.6.

- The sum of all elements of matrix $\breve{G}$ is one,

$$
\sum_{j=-\infty}^{\infty} S_{j}=1 \quad \text { or } \quad \sum_{j=-\infty}^{\infty} \sum_{k=-\infty}^{\infty} \check{G}_{j k}=1,
$$

since (62) and (30) for $\gamma=(1-2 \tau-\sqrt{1-4 \tau}) / 2 \tau$ give us

$$
\sum_{j=-\infty}^{\infty} \gamma^{|j|}=\frac{2}{1-\gamma}-1=\frac{1+\gamma}{1-\gamma}=\frac{1}{\sqrt{1-4 \tau}}
$$

\section{Appendix C Numerical implementation}

\section{C.1. High precision calculations}

In order to find elements $\breve{G}_{j k}$ one can use a finite sum in (61). However due to various rounding operations, there will be some additional error even if double precision numbers are used. Therefore it is better to try another approach. Formula (61) can be rewritten as $\check{G}_{j k}=(1-4 \tau) \tau^{j+k} \chi$,

$$
\chi=\sum_{q=0}^{\infty} a_{q} \tau^{2 q}, \quad a_{q}=C_{2 q+j+k}^{q} C_{2 q+j+k}^{q+j} .
$$

or a $\operatorname{sum} \chi=\chi_{1}+\chi_{2}$ of two terms

$$
\chi_{1}=\sum_{q=0}^{q^{\star}-1} a_{q} \tau^{2 q}, \quad \chi_{2}=\sum_{q=q^{\star}}^{\infty} a_{q} \tau^{2 q},
$$

where index $q^{\star}$ can be chosen later. The first term can be found using the recurrent formula

$$
\begin{aligned}
c_{q^{\star}} & =1, \\
c_{q} & =1+\frac{a_{q}}{a_{q-1}} \tau^{2} c_{q+1}, \quad q=q^{\star}-1, \ldots, 1
\end{aligned}
$$

and since $a_{0}=C_{j+k}^{0} C_{j+k}^{j}=C_{j+k}^{j}$, then $\chi_{1}=a_{0} c_{1}=C_{j+k}^{j} c_{1}$. Now we estimate from above the value of $\chi_{2}$. We get

$$
\frac{a_{q}}{a_{q-1}}=\frac{(2 q+j+k)^{2}(2 q+j+k-1)^{2}}{q(q+j)(q+k)(q+j+k)} .
$$

Note that $(q+j)(q+k) \geq q(q+j+k)$, so if denote $s=j+k$, we can estimate

$$
\frac{a_{q}}{a_{q-1}} \leq\left[\frac{(2 q+s)(2 q+s-1)}{q(q+s)}\right]^{2} .
$$

For $q \geq s(s-1) / 2$, we get

$$
\frac{a_{q}}{a_{q-1}} \leq 16
$$

and $\frac{a_{q}}{a_{q-1}} \tau^{2}<1$, so we can estimate from above the value of $\chi_{2}$

$$
\chi_{2} \leq a_{q^{\star}} \tau^{2 q^{\star}} \sum_{s=0}^{\infty}\left(16 \tau^{2}\right)^{s}=\frac{a_{q^{\star}} \tau^{2 q^{\star}}}{1-16 \tau^{2}} .
$$

As the result the value $\breve{G}_{j k}$ can be found by the following formula

$$
\begin{aligned}
& d_{q^{\star}}=\frac{1}{1-16 \tau^{2}}, \\
& d_{q}=1+d_{q+1} \frac{(2 q+j+k)^{2}(2 q+j+k-1)^{2}}{q(q+j)(q+k)(q+j+k)}, \quad q=q^{\star}-1, \ldots, 1, \\
& \check{G}_{j k}=(1-4 \tau) \tau^{j+k} C_{j+k}^{j} d_{1} .
\end{aligned}
$$

\section{C.2. Faster calculations}

Due to symmetry of $\check{G}_{j k}$ we have $\check{G}_{-1 k}=\check{G}_{1 k}$, so from equation (60) we obtain

$$
\check{G}_{1 k}=\frac{1}{2}\left(\frac{\check{G}_{0 k}}{\tau}-\check{G}_{0 k-1}-\check{G}_{0 k+1}\right) .
$$

The other rows can be found as

$$
\check{G}_{j k}=\frac{\check{G}_{j-1, k}}{\tau}-\check{G}_{j-1, k-1}-\check{G}_{j-1, k+1}-\check{G}_{j-2, k} .
$$

Acknowledgements The author would like to thank Albrecht Kyrieleis, Philip Withers and beamline scientists of Station 16.3 of Synchrotron Radiation Source (Daresbury Laboratory) and I12 beamline of the Diamond Light Source for the data provided.

\section{References}

Altunbas, C., Lai, C.-J., Zhong, Y. \& Shaw, C. C. (2014). Medical Physics, 41(9).

Baek, J., De Man, B., Harrison, D. \& Pelc, N. J. (2015). Optics Express, 23(6), 7514-7526.

Benjamin, A. T. \& Quinn, J. J. (2003). Proofs that really count. The art of combinatorial proof. Washington, DC: The Mathematical Association of America.

Brun, F., Accardo, A., Kourousias, G., Dreossi, D. \& Pugliese, R. (2013). In International Symposium on Image and Signal Processing and Analysis, ISPA, pp. 672-676.

Davis, G. R. \& Elliott, J. C. (1997). Nuclear Instruments and Methods in Physics Research, Section A: Accelerators, Spectrometers, Detectors and Associated Equipment, 394(1-2), 157-162.

Davis, G. R., Evershed, A. N. Z. \& Mills, D. (2013). Journal of Dentistry, 41(5), 475-482.

Drakopoulos, M., Connolley, T., Reinhard, C., Atwood, R., Magdysyuk, O., Vo, N., Hart, M., Connor, L., Humphreys, B., Howell, G., Davies, S., Hill, T., Wilkin, G., Pedersen, U., Foster, A., De Maio, N., Basham, M., Yuan, F. \& Wanelik, K. (2015). Journal of Synchrotron Radiation, 22, 828-838. 


\section{research papers}

Engl, H. W., Hanke, M. \& Neubauer, A. (1996). Regularization of inverse problems, vol. 375 of Mathematics and its Applications. Dordrecht: Kluwer Academic Publishers Group.

Gradshteyn, I. S. \& Ryzhik, I. M. (2015). Table of integrals, series, and products. Amsterdam: Elsevier/Academic Press.

Higham, N. J. (2008). Functions of Matrices: Theory and Computation. Philadelphia, PA, USA: Society for Industrial and Applied Mathematics.

Hinebaugh, J., Challa, P. R. \& Bazylak, A. (2012). Journal of Synchrotron Radiation, 19(6), 994-1000.

Kak, A. \& Slaney, M. (2001). Principles of Computerized Tomographic Imaging. Society for Industrial and Applied Mathematics.

Kalender, W. A. (2006). Physics in Medicine and Biology, 51(13), R29.

Miqueles, E. X., Rinkel, J., O’Dowd, F. \& Bermúdez, J. S. V. (2014). Journal of Synchrotron Radiation, 21(6), 1333-1346.

Münch, B., Trtik, P., Marone, F. \& Stampanoni, M. (2009). Optics Express, 17(10), 8567-8591.

Natterer, F. \& Wübbeling, F. (2001). Mathematical Methods in Image Reconstruction. Society for Industrial and Applied Mathematics.

Paleo, P. \& Mirone, A. (2015). Journal of Synchrotron Radiation, 22(5), 1268-1278.

Pešić, Z. D., Fanis, A. D., Wagner, U. \& Rau, C. (2013). Journal of Physics: Conference Series, 425(18), 182003.

Prell, D., Kyriakou, Y. \& Kalender, W. A. (2009). Physics in Medicine and Biology, 54(12), 3881-3895.

Rashid, S., Lee, S. Y. \& Hasan, M. K. (2012). Eurasip Journal on Advances in Signal Processing, 2012(1).

Sadi, F., Lee, S. Y. \& Hasan, M. K. (2010). Computers in Biology and Medicine, 40(1), 109-118.
Sijbers, J. \& Postnov, A. (2004). Physics in Medicine and Biology, 49(14), N247-N253.

Tikhonov, A. N. \& Arsenin, V. Y. (1977). Solutions of ill-posed problems. V. H. Winston \& Sons, Washington, D.C.: John Wiley \& Sons, New York-Toronto, Ont.-London.

Tikhonov, A. N., Goncharsky, A. V., Stepanov, V. V. \& Yagola, A. G. (1995). Numerical Methods for the Solution of Ill-Posed Problems, vol. 328 of Mathematics and Its Applications. Netherlands: Springer.

Titarenko, S., Titarenko, V., Kyrieleis, A. \& Withers, P. (2009). Applied Physics Letters, 95(7), 071113.

Titarenko, S., Titarenko, V., Kyrieleis, A. \& Withers, P. J. (2010a). Journal of Synchrotron Radiation, 17(4), 540-549.

Titarenko, S., Titarenko, V., Kyrieleis, A., Withers, P. J. \& De Carlo, F. (2011). Journal of Synchrotron Radiation, 18(3), 427-435.

Titarenko, S., Withers, P. J. \& Yagola, A. (2010b). Applied Mathematics Letters, 23(12), 1489-1495.

Titarenko, V. (2016). IEEE Signal Processing Letters, 23(6), 800-804.

Titarenko, V., Bradley, R., Martin, C., Withers, P. J. \& Titarenko, S. (2010c). In Developments in X-Ray Tomography VII, vol. 7804.

Titarenko, V., Titarenko, S., Withers, P. J., De Carlo, F. \& Xiao, X. (2010d). Journal of Synchrotron Radiation, 17(5), 689-699.

Warnett, J. M., Titarenko, V., Kiraci, E., Attridge, A., Lionheart, W. R. B., Withers, P. J. \& Williams, M. A. (2016). Measurement Science and Technology, 27(3), 035401.

Wei, Z., Wiebe, S. \& Chapman, D. (2013). Journal of Instrumentation, 8(6).

Wolkowski, B., Snead, E., Wesolowski, M., Singh, J., Pettitt, M., Chibbar, R., Melli, S. \& Montgomery, J. (2015). Journal of Synchrotron Radiation, 22(4), 1130-1138. 